\title{
Evaluating Treatment of Hepatitis C for Hemolytic Anemia Management
}

\author{
Swati DebRoy*,1 \\ Department of Mathematics, University of Florida, Gainesville, FL \\ Christopher Kribs-Zaleta \\ Department of Mathematics, University of Texas at Arlington, Arlington, TX \\ Anuj Mubayi \\ School of Human Evolution and Social Change, Arizona State University, Tempe, AZ / Department of Mathematics, \\ University of Texas at Arlington, Arlington, TX/ Prevention Research Center, Berkeley, CA/ Mathematical and \\ Computational Modeling Sciences Center, Arizona State University, Tempe, AZ \\ Gloriell M. Cardona-Meléndez \\ University of Puerto Rico, Cayey, Puerto Rico \\ Liana Medina-Rios \\ Mount Holyoke College, South Hadley, MA \\ MinJun Kang \\ Kyungpook National University, Daegu, Korea \\ Edgar Diaz \\ Mathematical and Computational Modeling Sciences Center, Arizona State University, Tempe, AZ
}

\begin{abstract}
The combination therapy of antiviral peg-interferon and ribavirin has evolved as one of the better treatments for Hepatitis-C. In spite of its success in controlling Hepatitis-C infection, it has also been associated with treatment-related adverse side effects. The most common and life threatening among them is hemolytic anemia, necessitating dose reduction or therapy cessation. The presence of this side effect leads to a tradeoff between continuing the treatment and exacerbating the side-effects versus decreasing dosage to relieve severe side-effects while allowing the disease to progress. The drug epoietin (epoetin) is often administered to stimulate the production of red blood cells (RBC) in the bone marrow, in order to allow treatment without anemia. This paper uses mathematical models to study the effect of combination therapy in light of
\end{abstract}

\footnotetext{
* Corresponding author

Email address: swati@math.ufl.edu (Swati DebRoy)

${ }^{1} 358$ Little Hall, PO Box 118105, Gainesville FL 32611-8105. Phone: (352) 392-0281*294 Fax: (352) 392-8357
} 
anemia. In order to achieve this we introduce RBC concentration and amount of drug in the body as state variables in the usual immunological virus infection model. Analysis of this model provides a quantification of the amount of drug a body can tolerate without succumbing to hemolytic anemia. Indirect estimation of parameters allow us to calculate the necessary increment in RBC production to be $\geq 2.3$ times the patient's original $\mathrm{RBC}$ production rate to sustain the entire course of treatment without encountering anemia in a sensitive patient.

Key words: Hepatitis-C, HCV, ribavirin, interferon, epoietin, anemia, hemolytic anemia, mathematical modeling.

Abbreviations: HCV, Hepatitis C, RBV, Ribavirin, peg-IFN, pegylated interferon, RBC, red blood cells, EPO, Epoietin, Epoetin.

\section{Introduction}

About one hundred and seventy million people live with Hepatitis C virus (HCV) infection world-wide [1]. Currently, there is no vaccine for HCV infection. The major mode of transmission of HCV is by exposure to infected blood. Sexual and vertical transmission of HCV has been reported; however, it is rare [2]. Hepatitis C (Hep-C) causes chronic diseases of the liver like cirrhosis and hepatocellular carcinoma [3]. The HCV infects hepatocytes which form a major portion of the cytoplasmic mass of the liver. Although HCV predominantly replicates in hepatocytes, traces of it have been detected in other cell types $[4,5]$.

Some patients with Hep-C infection will naturally clear the virus without medical intervention. However, a major proportion of HCV infected individuals develop chronic HCV infection in which the body's immune system does not naturally clear the virus. About 55 to $85 \%$ of HCV patients do not clear the virus themselves and develop chronic Hep-C infection [2]. The progression to chronic-stage HCV infection is a result of weak immune response against HCV (reviewed in [6]). Currently, the standard protocol for the treatment of Hep-C involves two antiviral drugs, Interferon- $\alpha$ (IFN) and ribavirin (RBV), given in combination $[7,8]$. It has been clinically observed that the combination of RBV and IFN demonstrates a synergistic pharmacological effect. That is to say, the treatment efficacy of this combination therapy goes well beyond the efficacies of the individual treatments added together [1]. Although the exact mechanism of the action of either of these drugs in treatment of Hep-C infection is not clear, direct antiviral, antiproliferative, and immunomodulatory activities of IFN are well known [1]. In the case of RBV, many scientists seem to favor its immunomodulatory 
action as an explanation of its efficacy in $\mathrm{HCV}$ treatment; however, its role as a viral RNA mutagen cannot be ignored, as discussed in Lau et al. and references therein [1]. Hep-C patients receive weekly injections of IFN and take RBV pills daily for the period of treatment [8]. If a patient does not show traces of Hep-C viral load in the body after six months of therapy cessation, the patient is said to have achieved Sustained Virological Response (SVR), implying clinically cured. The goal of this treatment is to lower the viral load and eventually achieve SVR. If SVR is not achieved, a patient is considered a chronic Hep-C patient. The rate of achievement of SVR in Hep-C patients varies according to the genotype of the particular infecting virus. The treatment for genotype 1 and 4 are usually carried out for 48 weeks and that for genotype 2 and 3 typically lasts for 24 weeks with lesser dosing of RBV [9]. Under this treatment, SVR rates of 46 percent are observed in patients with HCV genotype 1, whereas 76 percent and 82 percent of patients with HCV genotypes 2 or 3 achieve SVR [10].

In spite of being a sufficiently successful treatment for Hep-C infection, there are several negative side effects of the treatment ranging from flu-like symptoms and anemia to temporary disability and depression, which is a major cause for concern among medical practitioners. The most common and alarming side effect of IFN and RBV combination therapy is reversible hemolytic anemia necessitating dose reduction or complete cessation of RBV in many patients [11]. The RBV induces excessive hemolysis, that is, the breakdown of red blood cells $(\mathrm{RBC})$ and release of hemoglobin into the surrounding blood plasma. The body's ability to produce $\mathrm{RBC}$ at a faster rate to compensate for this excessive loss is stunted by the simultaneous bonemarrow suppressing effect of IFN [10]. The adjective 'reversible' signifies the fact that in most cases, the RBC count gets back to normal levels once the treatment is stopped. To combat the situation, doctors often prescribe a third drug, epoietin- $\alpha$ (EPO), which acts like the hormone erythropoietin and facilitates RBC production in the bone marrow [10]. Epoietin (also spelled epoetin) is observed to be quite efficient at reversing RBV-induced anemia in most patients. According to Sulkowski [10], success of the combination therapy with IFN and RBV is contingent on maintaining adequate doses of both drugs throughout the treatment period. The occurrence of side effects leads to a trade-off between continuing the treatment with optimal dosage to clear the virus and exacerbating the side effects versus decreasing dosage to relieve severe anemia, while reducing the chances of achieving SVR. With this in mind, we use mathematical modeling to estimate the EPO-induced increase in RBC production necessary for a patient to be able to undergo the complete course of the combination therapy without suffering from acute anemia.

The anti-anemic drug EPO is known to be well tolerated among HCV patients. However, increase in 
RBC production by more than a factor of 4 within 2 weeks should be avoided, and EPO does not increase RBC production without bounds for all patients [12]. Certain EPO-initiated side effects like pure red-cell aplasia due to presence of anti-erythropoietin antibodies has been observed in some patients with chronic renal failure $[13,10]$. Thus dosing only sufficient EPO is desirable.

In the context of Hep-C, mathematical modeling has been extensively used to determine the efficacy of IFN as monotherapy and in combination with RBV [7, 14]. Differences in response depending on genotype [15], and the concept of early virological response (EVR) to estimate possibility of achieving SVR [16] have been reinforced with mathematical models. Viral and drug kinetics studies using mathematical models have shed light on the understanding of this virus and its treatment in several directions [17]. All these studies have contributed enormously to the improvement of the treatment procedure that doctors presently apply to patients. We further this effort to develop mathematical methodologies that can be used to estimate the necessary usage of EPO to help an HCV patient to sustain the antiviral treatment.

We construct a set of coupled five ordinary differential equations where the first three represent uninfected target hepatocytes, infected hepatocytes and free virions based on the first model of Neumann et al.[18]. To that we incorporate the side effect of hemolytic anemia by considering the RBC concentration as a separate state variable and also the amount of drug in the body as a dynamic quantity rather than a constant parameter.

We focus on the interaction of the RBC level and amount of drug with the goal of finding an optimal drug treatment regimen to minimize the severity of anemia while still obtaining SVR. Using the first three equations we determine the minimum amount of drug necessary for the patient to achieve SVR. Then, we mathematically replicate a hypothetical situation with a sensitive Hep-C patient under combination therapy who starts taking the regular dosage prescribed by the doctors depending on body weight. Here, 'sensitive' means that the patient has a history of encountering hemolytic anemia when on this treatment. During treatment the patient's RBC concentration is monitored, and his dosage adjusted accordingly. From this process we calculate the amount of drug the patient can handle without hitting anemia. Then we introduce the effect of $\mathrm{EPO}$ in the equations, i.e., increase the RBC production by a factor of the patient's original $\mathrm{RBC}$ production level, to let the patient intake greater amounts of drug in the body while maintaining a healthy RBC concentration. This allows us to calculate the EPO-induced increment in RBC production necessary to let the patient sustain at least the minimum amount of drug to achieve SVR for the necessary period of time. A schematic representation of the usual scenario can be seen in Figure 1. Analytic and 


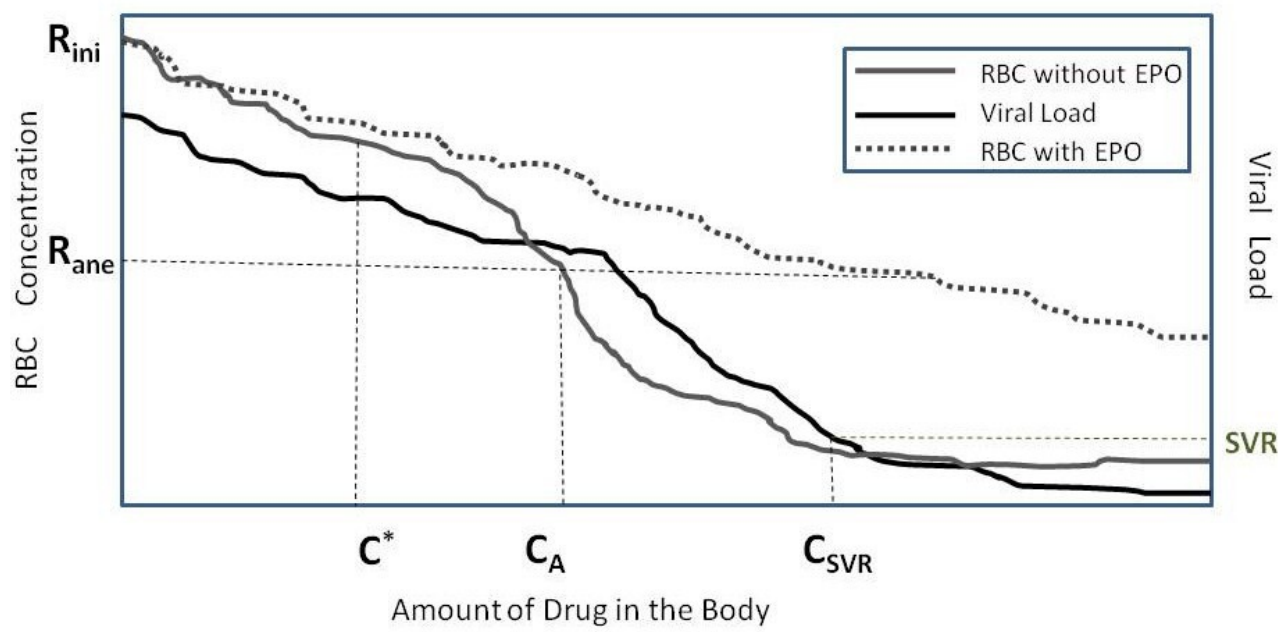

Figure 1: Schematic representation of sensitivity of RBC concentration in the body and viral load to the amount of drug in the body. The bold grey curve represents the RBC concentration in the patient which decreases as the amount of drug in the body increases. $R_{I}$ is the initial RBC concentration of the patient when no drug is present and $R_{A}$ is the RBC concentration at anemia. The black curve represents the viral load which also decreases as the amount of drug in the body increases. $C^{*}$ is the amount of drug in the body when the RBC concentration reaches equilibrium. $C_{A}$ is the amount of drug that causes anemia and $C_{S V R}$ is the minimum amount of drug required by the body to achieve SVR. $C^{*}, C_{A}, C_{S V R}$ are calculated from the model. A clinical problem occurs when the drug amount $C_{S V R}$ is much higher than $C_{A}$. Our goal is to use EPO to boost the RBC production to make $C_{A}$ greater than $C_{S V R}$. The grey dotted line represents the expected effect of EPO which keeps the RBC concentration at a healthy level even at higher doses of combination drugs. Further discussion of the drug related quantities can be found in Section 3.3.

numerical methods facilitate evaluation of several dosing regimens.

In Section 2, we introduce our model and its parameters; in Section 3 we analyze the model and calculate different reproductive numbers for $\mathrm{HCV}$ with and without treatment under varying assumptions. In Section 4 we discuss the estimation of parameters and establish the results of our analytic work numerically. In addition, critical drug amounts are numerically calculated for the system and the results of simulations are shown.

\section{Model}

Our model is an extension and modification of that used by Neumann et al. [18] which includes the state variables $T(t)$, target healthy hepatocytes; $I(t)$, infected hepatocytes; and $V(t)$, the viral load of free HCV. In order to focus on the interplay between the drugs and the RBC concentration in an HCV patient, we introduce two further state variables, $R(t)$ for the $\mathrm{RBC}$ concentration in the body and $C(t)$ for the amount of drug (IFN and RBV) in the body. (Note that $C(t)$ represents the total amount of drug in the body at 
time $t$ and not the concentration or dosage at time $t$.) We define $t=0$ to correspond to the beginning of treatment. Figure 2 illustrates the key elements of the model and their interactions (labeled by parameters).

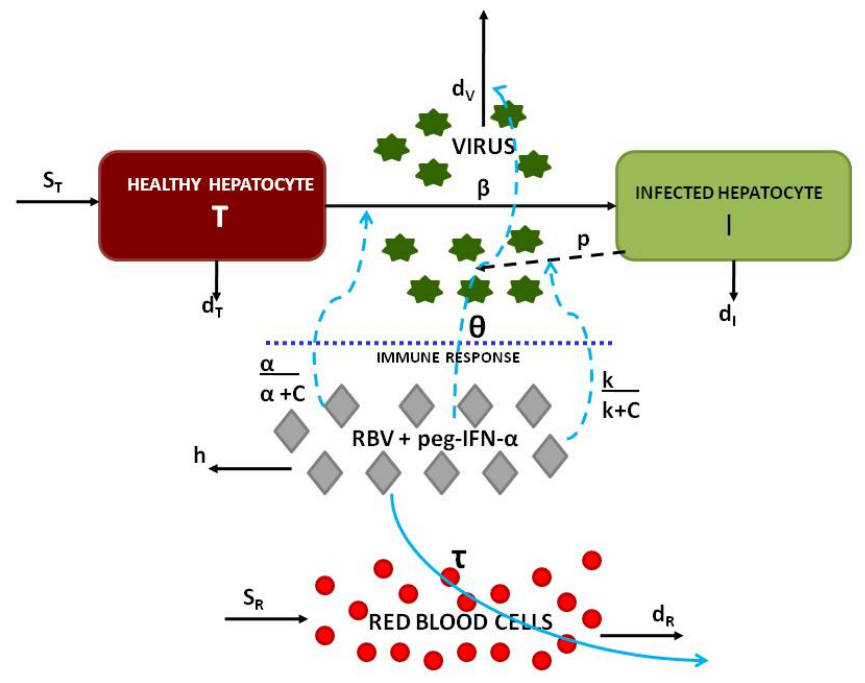

Figure 2: Compartmental Model

The model equations are:

$$
\begin{aligned}
\frac{d T}{d t} & =s_{T}-d_{T} T-\frac{\alpha}{\alpha+C} \beta T V \\
\frac{d I}{d t} & =\frac{\alpha}{\alpha+C} \beta T V-d_{I} I \\
\frac{d V}{d t} & =p \frac{k}{k+C} I-\theta C V-d_{V} V \\
\frac{d R}{d t} & =s_{R}-d_{R} R-\tau C R \\
\frac{d C}{d t} & =\Delta(R)-h C
\end{aligned}
$$

where

$$
\Delta(R)=\lambda \frac{R^{2}}{a^{2}+R^{2}}
$$

We begin by considering the drug-RBC interplay which is independent of the HCV within-host dynamics. We assume that the $\mathrm{RBC}$ concentration in the body $\left(R\right.$, measured in cells $\left.\mathrm{mL}^{-1}\right)$ is affected by three factors: the natural rate of $\mathrm{RBC}$ production in the bone marrow, which we denote by the constant $s_{R}$; natural cell 
mortality, which occurs at a rate $d_{R}$; and additional mortality induced by the presence of antiviral drugs in the body. Since this last rate is proportional to the amount of drug in the body, we model it with a mass action term $\tau R C$, where $\tau$ is the rate constant.

The amount of drug $C$ in the body, measured in mg, changes continuously over time in response to two factors: the drug dosage (in mg day ${ }^{-1}$ ), denoted by $\Delta(R)$, and the body's ability to clear the drugs from the body at a rate $h$ per day. A typical HCV patient is initially prescribed a dosage of IFN and RBV, based on his/her body weight. The daily RBV dosage is around 1000-1200 mg, and the IFN dosage is $0.1 \mu \mathrm{g}$ $\mathrm{kg}^{-1}$ week $^{-1}$, which is orders of magnitude lower in quantity. Although the pharmacokinetics and possibly modes of action of IFN and RBV are different, the mechanism of action of each of the drugs individually has not been firmly established, leaving the exact nature of their synergy even less confirmed [1]. Thus, for simplicity, rather than modeling the amount of each drug separately we consider the amount of drug $C$ to be the quantity of RBV since that is the drug which has the major effect on RBC levels and is reduced to half upon encountering anemia. However, we consider the efficacy of the drug to be that of the combination IFN-RBV therapy, since that is what affects the viral load.

We consider the dosage as a function of the RBC concentration, since in this scenario the goal is to avoid anemia caused by HCV therapy. If a patient encounters anemia at any point during the course of the therapy, doctors reduce the dosage of RBV to half, which increases the chances of unsuccessful achievement of SVR [10]. We are interested in finding out the maximum amount of drug a patient can take without encountering anemia. Thus, instead of waiting for the patient to hit anemia and then reducing the dosage to half we will consider the following hypothetical situation to construct our dosing term, $\Delta(R)$, in the $\frac{d C}{d t}$ equation. Suppose a Hep-C patient on combination drug therapy undergoes RBC concentration monitoring followed by a continuous reduction in the relative dose. Studying this correlation we can calculate the amount of drug in the body which keeps the RBC concentration at a healthy equilibrium. We first construct $\Delta(R)$, the dosage term as a function of RBC concentration, $R$. In reality, the dosage given by a doctor is best approximated by a RBC concentration dependent step function

$$
\Delta(R)=\left\{\begin{array}{cc}
\lambda & R>R_{A} \\
\frac{\lambda}{2} & R \leq R_{A}
\end{array}\right.
$$

where $\lambda$ is the initially prescribed dose and $R_{A}$ is the RBC concentration at anemia. However, in order to facilitate model analysis, here we construct a continuous function for our virtual dosing regimen to 
approximate the actual step function, according to the following properties:

1. it should be a strictly increasing function of $R$,

2. at time $t=0, \Delta(R)$ should equal the value of initial dosage $\lambda$ prescribed by the doctor, and

3. when the RBC concentration reaches anemic levels $\left(R=R_{A}\right) \Delta(R)$ should be reduced to half the original dosage, $\frac{\lambda}{2}$.

It can be noted that the function $\lambda \frac{R^{2}}{a^{2}+R^{2}}$ with its unique inflection point fits into criterion 1) very well. Using conditions 2) and 3) we calculate the constants $\lambda$ and $a$ in the numerical section. It is easy to note that $\lambda$ is the maximum possible dosage that can be theoretically administered. However, we never actually attain that, since as soon as we start treatment, the RBC concentration decreases, causing the entire dosage term to decrease. Also, the initial dosage will most likely be less than $\lambda$. Again, $a$ is the RBC concentration at which the dosage term $\Delta(R)$ is equal to half of $\lambda$.

We also suppose here that the patient's RBC concentration is constantly monitored and his dosage adjusted continuously. This assumption of optimal (continuous rather than discrete) response time permits the dosage adjustments to be incorporated into the qualitative analysis of the model, in addition to the numerical analysis. In practice the patient's condition is likely to be monitored once every several days, although in one clinical trial viral load was monitored several times per day [18]. This assumption also allows us to address the question of whether it is technically possible to achieve SVR while avoiding anemia.

This description of treatment as continuous in time and dosage is inaccurate in two regards. First, therapy is typically administered in discrete doses rather than continuously like some IV drugs. Second, in practice the dosage would not be adjusted on a continuous basis, but rather switched between a limited number (possibly only two) of levels, say the initial level and a reduced level. However, the first inaccuracy has minimal effect on the long-term predictions of the model, since doses are typically given daily anyway (see the end of Section 4.2 for an illustration of the difference). The second inaccuracy tends to idealize the effects of making drug dosage responsive to $\mathrm{RBC}$ concentration: that is, the model describes an optimal (immediate and continuous) responsiveness whereas real responsiveness is likely to be more coarse (in both time and RBC level). Thus the results evaluate "proof of concept" - whether the various drugs at their current efficiencies can be used in conjunction in such a way as to avoid anemia while achieving SVR. In order for a less adaptive, more uniform dosing policy to strike this critical balance, drug efficiencies (including EPO, which will be discussed later) will need to be higher than the threshold values calculated in this paper.

The $(\mathrm{T}, \mathrm{I}, \mathrm{V})$ system explains the HCV interaction in the hepatocyte cells of the liver. The parameter $s_{T}$ 
is the rate of production, and $d_{T}$ is the natural death rate of healthy hepatocytes from the bone marrow. $\alpha$ is related to the efficacy of combination therapy. It is essentially the amount of the drug at which production of new infected cells is reduced to half; that is, when $C=\alpha$, we get $\frac{\beta}{2}$. If there is no drug in the body (or the drug has no efficacy in reducing infection), $\frac{\alpha}{\alpha+C}=1$ and the virus infects the healthy hepatocytes at a constant rate $\beta$. If instead the drug works with $100 \%$ efficacy, $\frac{\alpha}{\alpha+C}=0$. $\beta$ is the number of infections caused by one infected cell per unit time. As the amount of drug increases, $\frac{\alpha}{\alpha+C}$ becomes a smaller fraction and the production of infected cells decrease. $\beta T V$ is the [unreduced] concentration (in cells $/ \mathrm{mL}$ ) of healthy hepatocytes infected by virus, $V$, per unit of time. This total rate of infected hepatocytes, $\beta T V$, goes into the second class of hepatocytes which is the infected hepatocytes, $I$. Here, $d_{I} I$ is the rate at which infected hepatocytes are cleared per unit of time.

The per capita production rate of $\mathrm{HCV}$ is $p$ and hence $p I$ accounts for the virions produced by the total population of infected hepatocytes per unit time. $k$, like $\alpha$, is related to the efficacy of the drugs. It is essentially the amount of the drug in the body which reduces the production of new virions to half of the amount produced in absence of treatment. $\theta$ is a rate with unit amount ${ }^{-1}$ time $^{-1} . \theta C V$ accounts for increased clearance of free virions due to the immunomodulatory effect of both drugs. Neumann et al. observed only a possibly minor effect of drugs on viral clearance in the first 2 days of therapy, based on a model which assumes constant drug efficacy over the whole period of treatment. Since, in our case, the efficacy of the drugs is constantly changing as RBC concentration varies, it is worth considering this possible effect of the drugs, in the absence of more extensive clinical data on this aspect of treatment. Since $d_{V}$ is the clearance rate of virion in absence of treatment, $d_{V} V$ is the total concentration of virions cleared per unit time.

\section{Analytical Work}

The model is essentially split into two separate systems because the RBC equation and drug amount equation are decoupled from the rest of the model. This allows us to analyze this model as two separate systems and as a cohesive system of equations. One of these subsystems is two-dimensional and considers only the effect that drug amount and RBC count have on each other. The other is three-dimensional and models the dynamics of target hepatocytes, infected hepatocytes, and free virions. 


\begin{tabular}{|c|l|}
\hline Parameter & Interpretation \\
\hline$s_{T}$ & natural production rate of hepatocytes \\
$s_{R}$ & natural production rate of RBC \\
$d_{V}$ & natural clearance rate of virus \\
$d_{T}$ & natural death rate of hepatocytes \\
$d_{I}$ & clearance rate of infected hepatocytes \\
$d_{R}$ & natural death rate of RBC \\
$\alpha$ & drug amount to reduce infection rate to half \\
$p$ & production rate of virus \\
$\beta$ & rate of new infections per virion \\
$k$ & drug amount to reduce virus production rate to half \\
$\theta$ & rate of virion death per unit drug \\
$\tau$ & rate of RBC death per unit drug \\
$\lambda$ & maximum drug dosage \\
$a$ & RBC concentration at which drug dosage is reduced to $\frac{\lambda}{2}$ \\
$h$ & rate of drug clearance \\
\hline
\end{tabular}

Table 1: This table gives a brief interpretation of the parameters used in the model.

\subsection{Analysis of $(\mathbf{R}, \mathbf{C})$ system}

We determine the equilibrium of the decoupled $\frac{d R}{d t}$ and $\frac{d C}{d t}$ without regard to the complete system. Equating $\frac{d C}{d t}=0$ we identify $C^{*}=\frac{\lambda}{h} \frac{R^{2}}{a^{2}+R^{2}}$. Then we use $C^{*}$ to find the abscissa of the equilibrium point, by equating $\frac{d R}{d t}$ to zero, resulting in the cubic equation

$$
F(R)=\left(d_{R}+\frac{\tau \lambda}{h}\right) R^{3}-s_{R} R^{2}+d_{R} a^{2} R-s_{R} a^{2}=0
$$

In light of this, we rescale the equation using $r=\frac{R}{a}, d=\frac{d_{R}}{S_{R} / a}$, and $\widetilde{a}=\frac{\tau \frac{\lambda}{h}}{S_{R} / a}$ to make our cubic equation depend on two parameters $\widetilde{a}$ and $d$ which simplifies to

$$
f(r)=(\widetilde{a}+d) r^{3}-r^{2}+d r-1=0
$$

Further details on these calculations can be found in Appendix A.1.

Observation 1. The equation (7) has exactly one positive real root.

Proof. Since $f(r)$ is cubic in $r$, there is a possibility of a maximum of 3 roots. Note that, if $r=0, f(r)<0$, and if $r$ is large, $f(r)>0$, so there is one or three real positive roots. Thus there cannot be 2 roots. Now let us consider the case of 3 possible roots. Using the rescaled equation, we analyze the possible roots in the parameter space. We consider $f(r)$ as a function of $\widetilde{a}$ and $d$, that is, $r^{3} \widetilde{a}+\left(r^{3}+r\right) d=r^{2}+1$. Then, we use 
the original equation and the first derivative of this equation $3 r^{2} \widetilde{a}+\left(3 r^{2}+1\right) d=2 r$. Since if $f(r)$ has two roots, $f(r)=0$ and at that point $f(r)$ should be tangent to the $x$-axis, thus $f^{\prime}(r)=0$. We apply linear algebra to this system of equations. Solving the system using the Gauss method, we find $\widetilde{a}=-\frac{\left(r^{2}+1\right)^{2}}{2 r^{3}}<0$ and $d=\frac{r^{2}+3}{2 r}>0$. These solutions are biologically extraneous since all parameter values are positive and the bifurcation lies only in the second quadrant as shown in Figure 3. Therefore, we have determined that the system does not have a biologically relevant bifurcation point.

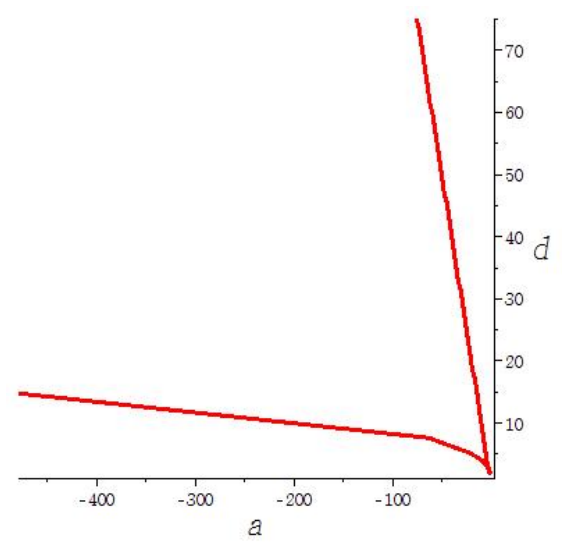

Figure 3: This curve is plotted using parametric curve $\widetilde{a}=-\frac{\left(r^{2}+1\right)^{2}}{2 r^{3}}<0$ and $d=\frac{r^{2}+3}{2 r}>0$

Hence, we conclude that the system cannot have three positive real roots. We determine that there is only one positive real root by analyzing the bifurcation diagram in the first quadrant. For further details on exact method, refer to Appendix A.2.

Observation 2. The equilibrium $\left(R^{*}, C^{*}\right)$ is locally asymptotically stable.

Proof. To analyze the local stability of this nontrivial equilibrium point we use a Jacobian matrix,

$$
J_{\left(R^{*}, C^{*}\right)}=\left[\begin{array}{cc}
-d_{R}-\tau C^{*} & -\tau R \\
2 \frac{a^{2} \lambda R}{\left(a^{2}+R^{* 2}\right)^{2}} & -h
\end{array}\right]
$$

Since $d_{R}>0, C^{*}>0, h>0, \tau>0$, we know $\operatorname{tr}(J)=-\left(d_{R}+\tau C^{*}+h\right)<0$, and $\operatorname{det}(J)=h\left(d_{R}+\tau C^{*}\right)+$ $\frac{2 \tau R^{* 2} a^{2} \lambda}{a^{2}+R^{* 2}}>0$, thus we show $\left(R^{*}, C^{*}\right)$ is locally asymptotically stable. For further details refer to Appendix A.3.

Observation 3. The equilibrium $\left(R^{*}, C^{*}\right)$ is globally stable 
Proof. We have proven local stability; now our interest is in the global stability of the (R,C) system. To this end, we apply the Poincaré-Bendixson Theorem. The Poincaré-Bendixson Theorem states that there are three possibilities for the asymptotic behavior of solutions to a two-dimensional system: a limit cycle, an unbounded solution or a stable equilibrium point. Now, using Dulac's criterion using the co-efficient function, $g=\frac{1}{R}$, we prove that there is no limit cycle. We also note by simple comparison that $\lim \sup _{t} C(t)<\frac{\lambda}{h}$ and $\lim \sup _{t} R(t)<\frac{s_{R}}{d_{R}}$; hence the solution cannot be unbounded. Thus local stability is extended to global stability for the unique equilibrium point. Further technical details are relegated to Appendix A.4.

Consequently by a theorem of Thieme [19] the behavior of the (1)-(5) system is asymptotic to the behavior of the three dimensional subsystem (1)-(3) with the equilibrium values $C^{*}$ and $R^{*}$ substituted for the state variables $C$ and $R$. In the next subsection, we will use this reduced system to determine the necessary equilibrium drug amount to eliminate $\mathrm{HCV}$ from the $(T, I, V)$ system.

\subsection{Analysis of $(\mathbf{T}, \mathbf{I}, \mathbf{V})$}

We analyze the $(T, I, V)$ subsystem and determine the desired critical drug amount, $C_{S V R}$, for which the disease free equilibrium of $(T, I, V)$ is stable. In the absence of Hepatitis $\mathrm{C}$ treatment, Callaway and Perelson [20] calculate the basic reproduction number $\left(\Re_{\mathrm{o}}\right)$.

$$
\Re_{\mathrm{o}}=\frac{1}{d_{I}} \frac{p \beta}{d_{V}} \frac{s_{T}}{d_{T}} .
$$

$\mathfrak{R}_{0}$ can be interpreted as the average number of newly infected T-cells produced by an infected T-cell during its (infected) lifetime. That is, an infected T-cell lives for $\frac{1}{d_{I}}$ units of time; each of $p$ virions produced by a bursting infected T-cell will produce $\frac{\beta}{d_{V}}$ new infected T-cells, from the population of uninfected T-cells of $\frac{s_{T}}{d_{T}}$.

We first analyze equations (1-3), taking $C$ as an asymptotic constant; in a sense we take it to be a parameter. Then the disease free equilibrium (DFE) is

$$
\left(T_{0}, I_{0}, V_{0}\right)=\left(\frac{s_{T}}{d_{T}}, 0,0\right)
$$

Linearizing the system about the DFE, and imposing conditions for stability we calculate $\widehat{\Re}$ which we call 
the controlled reproduction number $(\mathrm{CRN})$.

$$
\begin{aligned}
\widehat{\mathfrak{R}} & =\frac{\alpha}{\alpha+C} \frac{k}{k+C} \frac{d_{V}}{\theta C+d_{V}} \frac{s_{T} p \beta}{d_{I} d_{T} d_{V}} \\
\widehat{\mathfrak{R}} & =\frac{\alpha}{\alpha+C} \frac{k}{k+C} \frac{d_{V}}{\theta C+d_{V}} \mathfrak{R}_{\mathrm{o}}
\end{aligned}
$$

Observation 4. When $\widehat{\Re}<1$, the DFE is stable, and when $\widehat{\Re}>1$, the DFE exists but is unstable.

This observation is established in Appendix A.5. Thus, the infection is eliminated from the hepatocyte population when the DFE is stable. Now our attention shifts to the endemic equilibrium since our biological interest is treatment of chronically HCV infected individuals. Here the endemic equilibrium is defined as the equilibrium when $I^{*}>0$ and $V^{*}>0$. We solve the endemic equilibrium conditions to get:

$$
\begin{aligned}
T^{*} & =\frac{s_{T}}{d_{T} \widehat{\Re}} \\
I^{*} & =\frac{s_{T}}{d_{I}}\left(1-\frac{1}{\widehat{\Re}}\right) \\
V^{*} & =d_{T} \frac{(\alpha+C)}{\alpha \beta}(\widehat{\Re}-1)
\end{aligned}
$$

Observation 5. The endemic equilibrium of the $(T, I, V)$ system is globally stable if $\widehat{\mathfrak{R}}>1$.

See Appendix A.5 for further details on the equilibrium point of the (T,I,V) subsystem. The global stability of the $\left(T^{*}, I^{*}, V^{*}\right)$ equilibrium in the (T, I, V) subsystem can be proved using a Liapunov's function as in De Leenher and Pilyugin (2008) [21]. Defining the Liapunov's function as

$$
W=\xi \int_{T^{*}}^{T}\left(1-\frac{T^{*}}{\tau}\right) d \tau+\eta \int_{I^{*}}^{I}\left(1-\frac{I^{*}}{\tau}\right) d \tau+\zeta \int_{V^{*}}^{V}\left(1-\frac{V^{*}}{\tau}\right) d \tau
$$

and using properties of the equilibrium we have proved its global stability (see Appendix A.5.4).

\subsection{Critical Dosages}

Now that we have found the condition $(\widehat{\Re} \leq 1)$ for which the DFE is stable, we solve for $C_{S V R}$, the drug value for which $\widehat{\Re}=1$. This provides the minimum amount of drug needed to achieve SVR in a Hep-C patient. When we use equation (10) to solve for $C$, we obtain a cubic equation in $C$ of the form 
$G(C)=C^{3}+A_{2} C^{2}+A_{1} C+A_{0}=0$, where

$$
G(C)=C^{3}+\left(\frac{d_{V}}{\theta}+k+\alpha\right) C^{2}+\left(k \alpha+(k+\alpha) \frac{d_{V}}{\theta}\right) C+\left(k \alpha \frac{d_{V}}{\theta}\left(1-\mathfrak{R}_{\mathrm{o}}\right)\right) .
$$

We observe that $G(0)<0$ if and only if $\mathfrak{R}_{\mathfrak{o}}>1$. In addition $G^{\prime}(C)>0$ for each $C$ and $\lim _{C \rightarrow \infty} G(C)=+\infty$; therefore there exists one unique root, namely

$$
C_{S V R}=-A_{2}-\frac{1}{3 \sqrt[3]{2}}\left[\left(B_{1}+\sqrt{B_{1}^{2}-4 B_{2}}\right)^{\frac{1}{3}}+\left(B_{1}-\sqrt{B_{1}^{2}-4 B_{2}}\right)^{\frac{1}{3}}\right]
$$

where

$$
\begin{aligned}
& B_{1}=2 A_{2}^{3}-9 A_{1} A_{0}+27 A_{0} . \\
& B_{2}=A_{2}^{2}-3 A_{1} .
\end{aligned}
$$

It has been noted in [18] that for higher efficacy of the therapy, the effect of treatment on the infection rate $\beta T V$ is negligible. In this limiting case we have $\frac{\alpha}{\alpha+C}=1$. Then equation (14) becomes quadratic:

$$
C^{2}+\left(k+\frac{d_{V}}{\theta}\right) C+k \frac{d_{V}}{\theta}\left(1-\Re_{\mathfrak{o}}\right)=0
$$

with solution

$$
C_{S V R}=\frac{-\left(d_{V}+\theta k\right)+\sqrt{\left(d_{V}+\theta k\right)^{2}+4 k d_{V}\left(\Re_{\mathfrak{o}}-1\right)}}{2 \theta} .
$$

Note that we introduce treatment into an infected population only if $\mathfrak{R}_{\mathfrak{o}}>1$, implying that the body is incapable of clearing out the virus by itself. Therefore, $\left(\Re_{\mathrm{o}}-1\right)>0$ making $C_{S V R}>0$ always.

As mentioned before we define $C_{A}$ as the maximum amount of drug a body can tolerate without encountering anemia. To compute $C_{A}$ we first determine $R_{A}$, i.e., the $\mathrm{RBC}$ concentration at anemia for the patient, which is a clinically fixed quantity independent of the amount of drug in the body. Equation (5) represents the change in $\mathrm{RBC}$ concentration with respect to the amount of drug, and at equilibrium it gives a relationship between the amount of drug in the body and the $\mathrm{RBC}$ concentration. Thus, using the RBC concentration $R_{A}$ we solve $d R / d t=0$ for $C_{A}$, the amount of drug (IFN+RBV) in the body that causes 
anemia in the absence of additional treatment with EPO:

$$
C_{A}=\frac{s_{R}-d_{R} R_{A}}{\tau R_{A}}
$$

Thus, if the amount of drug in the body is more than $C_{A}$, a person will get anemia. In terms of the biology of Hepatitis $\mathrm{C}$ treatment and the side effect of anemia, the analysis of the $(R, C)$ and $(T, I, V)$ subsystems allows us to compare the following critical drug amounts: the equilibrium drug amount $C^{*}$, which keeps the $\mathrm{RBC}$ concentration at an equilibrium; the minimum amount of drug in the body so that the viral load goes below detection, $C_{S V R}$, leading to successful achievement of SVR; and the drug amount $C_{A}$, that is, the maximum amount of drug a patient can tolerate without getting anemia. Numerical estimation of these values, and their relative values can provide an objective view about the patient's condition and necessary steps to be taken. This is explained in detail in the results, Section 5 .

The ideal scenario for a doctor is to observe $C_{S V R} \leq C_{A}$. Then depending upon other factors concerning the patient he can prescribe a dosing regime so that the amount of drug in the body stays in the interval $\left(C_{S V R}, C_{A}\right)$. That will ensure that SVR is achieved without encountering anemia. The patients for whom the inequality is reversed, we treat with EPO to boost $C_{A}$ higher than $C_{S V R}$ so that doctors have an opportunity to treat them without anemic complications.

To introduce the effect of epoietin we increase (multiply) the RBC production by a factor $b$ and recalculate $C_{A}$. Epoietin is known to increase RBC production by a factor of the patient's original RBC production level from clinical trials [10]. The aim for our treatment is to achieve $C_{S V R} \leq C_{A}$ by increasing the production of RBC in equation (17) by a factor of $b(b>1)$. Solving for $b$ in

$$
C_{S V R}=\frac{b s_{R}-d_{R} R_{A}}{\tau R_{A}}
$$

calculates the critical factor $b_{c}$ which is the minimum-fold increase in RBC production using epoietin for a particular patient, to safely achieve SVR. In the following section, we estimate the value of $b_{c}$ from numerical simulations. 


\section{Numerical Simulation}

\subsection{Model Parameter Estimation}

In this section we address the estimation of some of the parameters using various sources and 'indirect' estimation procedures in the absence of real patient data. Please note that this numerical calculation is more of a 'proof of idea' and not an effort to estimate the dosing for every patient. All the assumptions and special cases considered in this section are mentioned in the discussion of estimation of individual parameters. During estimation, we fix the values of certain parameters $\left(s_{T} ; d_{T} ; d_{I} ; d_{R} ; p ; \beta\right.$ and $\left.h\right)$ taken from literature (given in Table 3) [7], [22] and [23]. Recall that our goal is to provide optimal treatment for a HCV patient who otherwise would develop anemia under standard treatment. In this theoretical study, we capture this hypothetical HCV patient by appropriately estimating the rest of the parameters (except $b$ ). We apply the effect of EPO (anti-anemic drug) to this patient, through change of the parameter $b$ in the model. The complete list of parameters including the estimated ones is tabulated in Table 3 .

\section{Estimating $\lambda$ and $a$}

We first develop estimates for parameters $\lambda$ and $a$ used in the dosing function $\Delta(R)$. As stated in Section 2 , this function should have the property that when the RBC concentration is normal, say $R=R_{I}$ (as at the onset of treatment), $\Delta\left(R_{I}\right)$ gives the initial dosage $\Delta_{I}$ prescribed by a doctor, and when anemia occurs, $R=R_{A}$, the dosage is cut in half, $\Delta\left(R_{A}\right)=R_{I} / 2$. If $R_{I}, R_{A}$, and $\Delta_{I}$ are known, then we can solve these equations,

$$
\Delta_{I}=\lambda \frac{R_{I}^{2}}{a^{2}+R_{I}^{2}} \text { and } \Delta_{I} / 2=\lambda \frac{R_{A}^{2}}{a^{2}+R_{A}^{2}}
$$

to obtain $\lambda$ and $a$ :

$$
a=\frac{R_{I} R_{A}}{\sqrt{R_{I}^{2}-2 R_{A}^{2}}}=\frac{1}{\sqrt{\frac{1}{R_{A}^{2}}-\frac{2}{R_{I}^{2}}}} \text { and } \lambda=\Delta_{I}\left(1+\frac{a^{2}}{R_{I}^{2}}\right)=\Delta_{I} \frac{R_{I}^{2}-R_{A}^{2}}{R_{I}^{2}-2 R_{A}^{2}}
$$

Doses of RBV are typically prescribed based on hemoglobin concentration at a given point in time. It is known from [24] that the RBC-to-hemoglobin ratio in a person is roughly constant, as makes biological sense. Therefore, taking values for $R_{I}$, the usual (initial) hemoglobin concentration $H_{I}$, and the hemoglobin concentration $H_{A}$ at anemia from [25], we can determine the RBC concentration $R_{A}$ at anemia by a simple proportion:

$$
\frac{R_{I}}{R_{A}}=\frac{H_{I}}{H_{A}} .
$$


We take the value of $\Delta_{I}=1200 \mathrm{mg}$ day $^{-1}$ for the initial dosage from [10]. The clinical definition of anemia appears to vary in the literature: papers like Afdhal et al. reduce dosing of RBV at hemoglobin level $<12$ $\mathrm{g} \mathrm{dL}^{-1}[11]$ whereas Sulkowski et al. take this value to be $10 \mathrm{~g} \mathrm{dL}^{-1}$ [10]. In this paper we use $10 \mathrm{~g} \mathrm{dL}^{-1}$ as the hemoglobin level at which the RBV dose is reduced to half. From these quantities we can then use equations (19) to calculate $\lambda$ and $a$ (see Table 4.1 for all values).

Table 2: Base values used to determine dosing parameters

\begin{tabular}{|c|l|lc|}
\hline Symbol & Meaning & Value & Source \\
\hline$H_{I}$ & Normal (initial) hemoglobin level & $160 \mathrm{mg} \mathrm{mL}^{-1}$ & {$[25]$} \\
$H_{A}$ & Hemoglobin level at anemia & $100 \mathrm{mg} \mathrm{mL}^{-1}$ & {$[25]$} \\
$R_{I}$ & Normal (initial) RBC concentration & $6.1 \times 10^{3}$ cells mL$^{-1}$ & {$[25]$} \\
$R_{A}$ & RBC concentration at anemia & $3.8 \times 10^{3}$ cells mL $^{-1}$ & eqn. (20) \\
$\Delta_{I}$ & Initial dosage & $1.2 \times 10^{3} \mathrm{mg} \mathrm{day}^{-1}$ & {$[10]$} \\
\hline$a$ & Half-saturation constant for dosing & $8.1 \times 10^{3} \mathrm{cells} \mathrm{mL}^{-1}$ & eqn. (19) \\
$\lambda$ & Maximum dosage & $3.3 \times 10^{3} \mathrm{mg} \mathrm{day}^{-1}$ & eqn. (19) \\
\hline
\end{tabular}

It should be noted that equations (19) (and hence the criteria from which they were derived) require that $R_{I} / R_{A}>\sqrt{2} \approx 1.414$, that is, that the normal $\mathrm{RBC}$ concentration be at least $41.4 \%$ greater than the $\mathrm{RBC}$ concentration at anemia. From (20), this is equivalent to saying $H_{I} / H_{A}>\sqrt{2}$. If this inequality does not hold, then it is not possible for a dosing function of the given form (a rational quadratic) to obey the constraint $\Delta\left(R_{A}\right)=\Delta\left(R_{I}\right) / 2$, and one must adjust either the function (to, say, a rational polynomial of higher order) or the constraint. In clinical practice, an anemic patient is often given medication to boost up the hemoglobin count to healthy levels before antiviral therapy is initiated. Since $H_{A}$ is always $100 \mathrm{mg}$ $\mathrm{mL}^{-1}$, the initial hemoglobin level of $140 \mathrm{mg} \mathrm{mL}^{-1}$ is desired to apply this model. This hemoglobin level could be too much to ask from a patient with history of anemia since hemoglobin levels of approximately 120-150 mg mL $\mathrm{mL}^{-1}$ for women, $130-170 \mathrm{mg} \mathrm{mL}^{-1}$ for men, are considered normal.

\section{Estimation of $s_{R}$}

In a healthy or uninfected individual, the dynamics of the RBC concentration will be

$$
\frac{d R}{d t}=s_{R}-d_{R} R
$$

Then at equilibrium, $s_{R}=d_{R} R$. From [22], we get that $d_{R}=.0231$ day $^{-1}$. Now using the initial RBC concentration $R=R_{I}$ (given in Table 4.1), we calculate $s_{R}=1.4 \times 10^{2}$ cells $\mathrm{mL}^{-1}$ day $^{-1}$.

\section{Estimation of $\tau$}


We estimate the value of $\tau$ using the dynamical equation $\frac{d R}{d t}$ by substituting appropriate estimates of $R, C, \frac{d R}{d t}$ along with the parameter values, as follows.

$$
\tau=\frac{1}{R C}\left(s_{R}-d_{R} R-\frac{d R}{d t}\right)
$$

Note that

$$
\frac{d R}{d t} \approx \frac{R_{I}-R_{A}}{\delta t}
$$

where $\delta t$ is the time taken for the initial RBC concentration to decrease to anemic levels.

From the results of the clinical trial in [26], we know that more than $50 \%$ of patients receiving IFN- $\alpha$ and RBV encountered anemia within the 14-28 days leading to dose reduction. From Table 4.1, we see that our hypothetical patient will develop anemia if the reduction in RBC concentration is $R_{I}-R_{A}=2.3 \times 10^{3}$ cells $\mathrm{mL}^{-1}$. Since our patient is 'sensitive', we assume this reduction to take place within 14 days of initiation of therapy. Hence, $\frac{d R}{d t} \approx-\frac{2.3 \times 10^{3}}{14}$ cells $\mathrm{mL}^{-1}$ day $^{-1}$.

To calculate the amount of drug in the expression for $\tau$, we consider the effect of drug on the RBC concentration if a constant dosing regime was used instead of the 'self-adjusting' dosing. This dynamics is represented by the equation $\frac{d C}{d t}=\Delta_{I}-h C$. Then at equilibrium, the amount of drug in the body would be

$$
\begin{aligned}
C_{\text {avg }} & =\frac{\Delta_{I}}{h} \\
& =631.5 \mathrm{mg}
\end{aligned}
$$

Since the patient encounters anemia at $t=14$ days, we take the RBC concentration $R=R_{A}$. Substituting these values we calculate $\tau=9 \times 10^{-5} \mathrm{mg}^{-1}$ day $^{-1}$.

From the last two equations, i.e., from the $\frac{d R}{d t}=0$ and $\frac{d C}{d t}=0$ equations we calculate the $C^{*}=233.67$ $\mathrm{mg}$ and $R^{*}=3.19 \times 10^{3}$ cells $\mathrm{mL}^{-1}$.

\section{Estimation of $\alpha$ and $k$}

Neumann et al. [18] found that the initial drop in the viral load under therapy depends mostly on the efficacy of the drug on the production rate of virus. In fact, if the efficacy of the drug on $p$ is $100 \%$, then the effect on $\beta$ can be ignored. However, no drug is perfect, and if $\frac{k}{k+C}<1$, the effect of drug on $\beta$ becomes more prominent as treatment continues. Other literature has modeled treatment of HCV with these antivirals without considering any effect of drugs on $\beta$ at all $[14,27]$. 
Here we perform our primary computations considering the scenario that there is no effect of drugs on $\beta$. The changes in final result due to considering the effect of drugs with $50 \%$ efficacy in reducing infection $\left(\frac{\alpha}{\alpha+C}\right)$ will be discussed in the results section. Now, $\frac{k}{k+C}$ is the efficacy of the combination therapy in reducing new virion production in infected cells. From Herrmann et al. [28] we have that the mean efficacy of the therapy with standard IFN and RBV is $36 \%$ and of peg-IFN is $63 \%$. Taking the average we use $\epsilon=49 \%$, for the calculation of $k$. For the amount of drug in the expression for $k$ we use the value of $C_{a v g}$ as calculated in equation 21.

Therefore,

$$
\begin{aligned}
\frac{k}{k+C} & =(1-\epsilon) \\
k & =\frac{1-\epsilon}{\epsilon} C \\
k & =657 \mathrm{mg} .
\end{aligned}
$$

\section{Estimation of $\theta$ and $d_{V}$}

The average clearance rate of free HCV in Dahari et al. [7] is 7.8 per day which is estimated in the presence of drugs. It is difficult to find the clearance rate of the virus in vivo in the absence of treatment. Most patients who are diagnosed for HCV infection get treated immediately. Here, for illustration purposes, we investigate the scenario where there is a $30 \%$ increase in clearance rate due to the antivirals. Then we have $d_{V}+\theta C=7.8$ day $^{-1}$. Assuming a $30 \%$ increase due to the drug, we should have a pre-treatment clearance rate $d_{V}=7.8-30 \%=5.5 \mathrm{day}^{-1}$. Thus, $\theta C=2.3 \mathrm{day}^{-1}$. For the amount of drug $C$ we use the value of $C^{*}$ due to reasons explained at the end of section 2. Note that at this point we have already estimated all the parameters necessary to calculate $C^{*}$. This gives $\theta=0.009 \mathrm{mg}^{-1}$ day $^{-1}$. The effect on the numerical result if we assume $\theta=0$ is discussed in section 4.2 .

\section{Calculation of Initial Conditions}

The ( $\mathrm{T}, \mathrm{I}, \mathrm{V})$ equations at $C=0$ give us the dynamics of the $\mathrm{HCV}$ virus in the patient before initiation of treatment. We calculate the equilibrium values of the variables in this system and use these as the initial condition for T, I and V in our model with treatment. Thus,

$$
\begin{aligned}
(T(0), I(0), V(0)) & =\left(\frac{d_{I} d_{V}}{p \beta}, \frac{d_{V} V(0)}{p}, V(0)\right) \\
& =\left(2.19 \times 10^{6}, 1.8 \times 10^{6}, 10^{6}\right),
\end{aligned}
$$


Table 3: Parameter Value Table

\begin{tabular}{|c|c|c|}
\hline Parameter & Value & Reference \\
\hline$s_{T}$ & 26000 cells $\mathrm{mL}^{-1} \mathrm{day}^{-1}$ & {$[7]$} \\
\hline$s_{R}$ & $1.4 \times 10^{2}$ cells $\mathrm{mL}^{-1} \mathrm{day}^{-1}$ & estimated * \\
\hline$d_{V}$ & $5.5 \mathrm{day}^{-1}$ & estimated * \\
\hline$d_{T}$ & $0.0026 \mathrm{day}^{-1}$ & {$[7]$} \\
\hline$d_{I}$ & $0.26 \mathrm{day}^{-1}$ & {$[7]$} \\
\hline$d_{R}$ & $.0231 \mathrm{day}^{-1}$ & {$[22]$} \\
\hline$p$ & 2.9 virions cells ${ }^{-1}$ day $^{-1}$ & {$[7]$} \\
\hline$\beta$ & $2.25 \times 10^{-7} \mathrm{~mL}$ virion $^{-1}$ day $^{-1}$ & {$[7]$} \\
\hline$k$ & $657 \mathrm{mg}$ & estimated * \\
\hline$\theta$ & $.009 \mathrm{mg}^{-1} \mathrm{day}^{-1}$ & estimated * \\
\hline$\tau$ & $9 \times 10^{-5} \mathrm{mg}^{-1} \mathrm{day}^{-1}$ & estimated * \\
\hline$\lambda$ & $3.3 \times 10^{3} \mathrm{mg} \mathrm{day}^{-1}$ & estimated * \\
\hline$a$ & $8.1 \times 10^{3}$ cells $\mathrm{mL}^{-1}$ & estimated * \\
\hline$h$ & 1.9 day $^{-1}$ & {$[23]$} \\
\hline
\end{tabular}

where $V(0)=10^{6}$ is derived from [7].

The initial condition $R(0)=R_{I}$ as mentioned before and $C(0)=0 \mathrm{mg}$, as time $t=0$ corresponds to initiation of therapy.

Table 4: Initial Conditions of State Variables
\begin{tabular}{|c|l|}
\hline Variable & Initial Value \\
\hline$T(0)$ & $2.19 \times 10^{6}$ cells $\mathrm{mL}^{-1}$ \\
$I(0)$ & $1.8 \times 10^{6}$ cells $\mathrm{mL}^{-1}$ \\
$V(0)$ & $1 \times 10^{6}$ virion $\mathrm{mL}^{-1}$ \\
$R(0)$ & $6.1 \times 10^{3}$ cells $\mathrm{mL}^{-1}$ \\
$C(0)$ & $0 \mathrm{mg}$ \\
\hline
\end{tabular}
Initial values of variables used for the simulation.

\subsection{Numerical Results}

Recall that solving the equilibrium equations $\frac{d R}{d t}=0$ and $\frac{d C}{d t}=0$ numerically we calculate the steady state RBC concentration, $R^{*}=3.19 \times 10^{3}$ cells $\mathrm{mL}^{-1}$, which is much below the anemic level of RBC concentration, calculated above as $3.8 \times 10^{3}$ cells $\mathrm{mL}^{-1}$. From equation (16), we get that $C_{S V R}=691 \mathrm{mg}$. That is, the minimum amount of drug that should be present in the body on an average, to ensure achievement of SVR is $691 \mathrm{mg}$. The maximum drug amount that the body can take in and still avoid anemia, calculated from (17), is $C_{A}=153 \mathrm{mg}$. Here, the minimum amount of drug necessary for a patient to achieve SVR is greater than the maximum amount of drug a patient can tolerate without encountering anemia, i.e., $C_{A}<C_{S V R}$, a 
common clinical scenario, which is a cause for concern among doctors, as mentioned earlier. To address this situation, doctors administer EPO. The aim of the treatment would be to boost $C_{A}$ so that $C_{S V R} \leq C_{A}$, where the patient gets cured while not getting anemia in the entire period of treatment. From equation 18, we get that, to achieve $C_{A}=C_{S V R}$ the factor $b=b_{c}=2.31$. That is, enough epoietin is needed to boost the $\mathrm{RBC}$ production by around 2.3 times. Figure 4 shows the increase in $C_{A}$ as EPO increases RBC production in the body by increasing $b$.

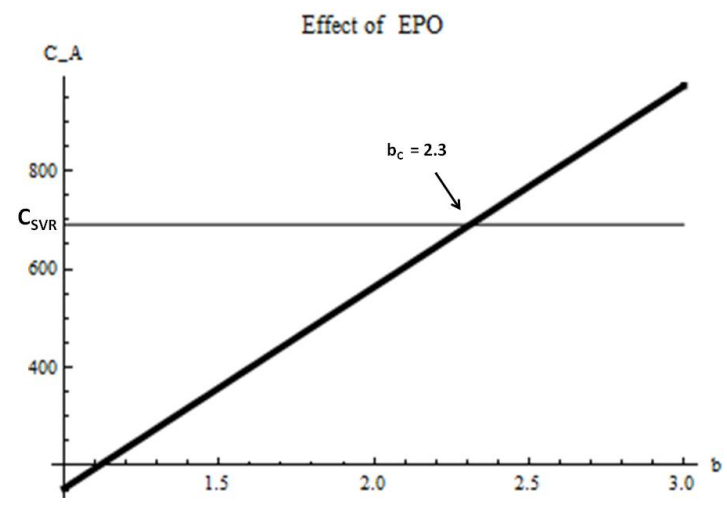

Figure 4: In this graph the thick line shows the increase of $C_{A}$ as EPO increases the production of RBC in the body. The thin horizontal line represents the drug amount $C_{S V R}$. Therefore the critical factor $b_{c}$ is marked by the intersection of these two lines, as indicated by the arrow.

Thus we conclude that RBC production in this particular patient has to be increased by more than 2.3 times, to sustain a continuous dosage of $1200 \mathrm{mg}$ per day along with normal RBC concentration for the entire period of treatment around 48 weeks $=336$ days. It might appear that the more we increase $\mathrm{RBC}$ production the better the result. However, the ability of EPO to increase RBC production is limited, as mentioned earlier [12]. A HCV patient has been observed to increase RBC production by a maximum of 3 times only [10]. Many patients with weak immune system do not respond well to EPO dosing, as explained in the introduction. Thus the minimum increment necessary to endure antiviral treatment should be targeted.

We numerically solve the entire model (1)-(5) without and with epoietin at different levels, for the period of 48 weeks treatment (=336 days). For the purpose of this paper we are interested in the period of treatment only, since therapy-induced hemolytic anemia is reversible. That is, RBC concentration shoots back to healthy levels as soon as these antivirals are discontinued. We note in the graphs in Figure $5 \& 6$, when EPO is not administered (denoted by bold line), the RBC concentration drops below anemic levels. Due 
to that, our hypothetical dosing regimen starts decreasing the antiviral dosing, leading to a relapse in viral load even before the end of the treatment period. When instead we give enough EPO to increase the RBC production by 2.3 times (denoted by large dashed line), the viral load is significantly reduced throughout the period of therapy whereas the RBC concentration is maintained at non-anemic levels throughout treatment. Note that the RBC can also be maintained at non-anemic levels (dotted line) when a lighter dose of EPO (causing approximately 1.5 times increase in RBC production) is given. However, the viral load starts to relapse around therapy cessation (336 days), resulting in unsuccessful achievement of SVR. (In practice, relapse is more common after treatment stops than before.) Finally, a higher dose of EPO to increase RBC production by 3 times (small dashed line) can maintain the RBC concentration at healthy pre-treatment levels, which helps the body sustain a higher constant concentration of the antivirals. Although this appears to be the perfect scenario, it is practically almost impossible to increase a person's RBC production by 3 times without significant complications, as described before.

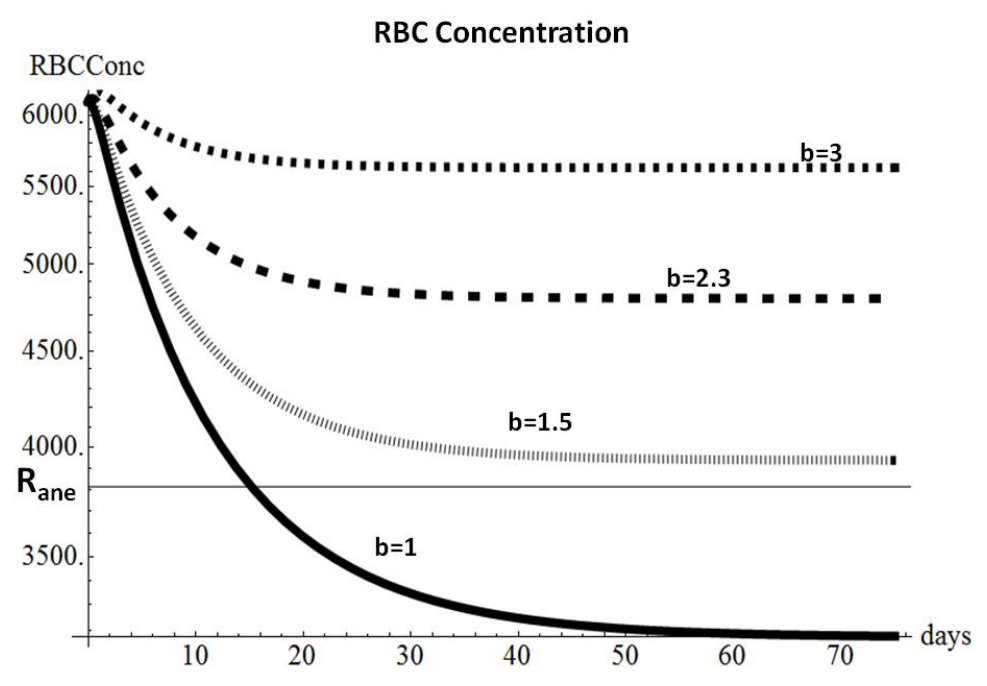

Figure 5: In this graph we observe the trajectories of the RBC concentration at different levels of EPO administration. The bold line shows the trajectory when no EPO is give, i.e. $b=1$. The small dashed lines show the behavior when only EPO enough to make $C_{A}=C_{S V R}$ is given to a patient. When enough EPO to increase RBC production about 1.5 times is given, we note from the dotted line that the RBC concentration is maintained above anemic levels. The large dashed line shows the behavior of the state variable when sufficient EPO is given to achieve $b=3$. We observe that the viral load has a steep decline but does not shoot back during the period of treatment of 48 weeks $=336$ days (see Figure 6 ), and the RBC concentration is maintained at pre-treatment levels. However, this might be clinically undesirable.

Because estimates for several model parameters are based on scant data and/or heuristic arguments, we have carried out uncertainty analysis on $C_{S V R}$ and $R_{0}$ as a function of some of the input parameters 
(see Figure 7(a)). The mean estimates and ranges of the input parameters are obtained from the literature $([18,28])$. The parameter $\epsilon$ (used in estimation of $k$ and $\alpha$ ) is assumed normally distributed with mean 0.36 and variance 0.09 whereas parameters $d_{V}, d_{I}$ and $R_{I}$ are assumed uniformly distributed with mean 6.20 day $^{-1}, 0.24$ day $^{-1}$ and 5450 cells $\mathrm{mL}^{-1}$, respectively. Distributions for the rest of the input parameters $\left(R_{A}\right.$, $\left.a, \lambda, s_{R}, \tau, k\right)$ are derived using model equations. The distributions for unknown parameters are obtained via a Monte-Carlo sampling procedure. Variation in $\lambda$ (mean $=3342 \mathrm{mg} /$ day) and $C_{A}$ (mean=154mg) are found to be negligible. On the other hand, relatively high variance is found in our output variables $C_{S V R}$ (mean=786 mg, $\mathrm{SD}=153 \mathrm{mg}$ ) and $R_{0}($ mean $=5.03, \mathrm{SD}=2.01$ ).

In order to determine which model parameter(s) may have the most effect on these critical quantities, we carried out a sensitivity analysis on $C_{S V R}$, a function of parameters $k, \theta, s_{T}, \beta, p, d_{T}, d_{V}$ and $d_{I}$. The local sensitivity indices (SI) are computed by normalizing the partial derivatives of $C_{S V R}$ with respect to each of its parameters. The SI suggest that the most sensitive parameter to $C_{S V R}$ is $\theta$ followed by $\beta$ whereas the least sensitive among its parameters is $k$ (see Figure $7(\mathrm{~b})$ ). $C_{S V R}$ is positively related to parameters $k, s_{T}$, $\beta$ and $p$, whereas $\theta, d_{T}, d_{V}$ and $d_{I}$ are negatively related. This implies that increases in the former set of

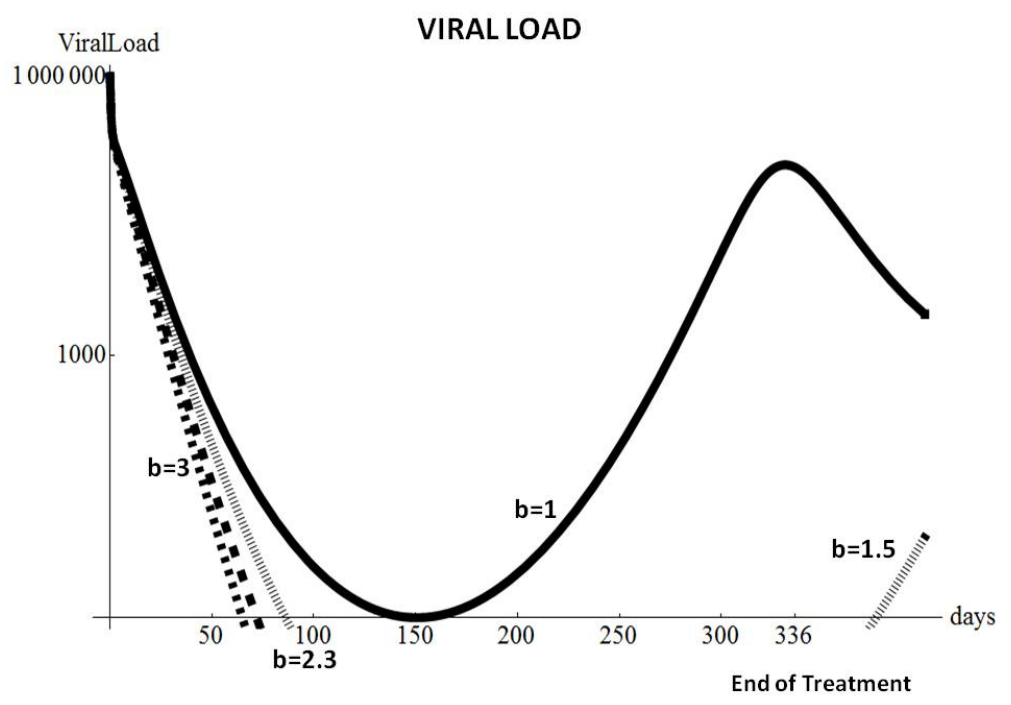

Figure 6: In the above graph, we show by the bold line the dynamics of the viral load. The small dashed lines shows the behavior when only EPO enough to make $C_{A}=C_{S V R}$ is given to a patient. When enough EPO to increase RBC production about 1.5 times is given, we note from the dotted line that the viral load tends to re-emerge before therapy cessation although the RBC concentration is maintained above anemic levels (see Figure 5). The large dashed line shows the behavior of the viral load when sufficient EPO is given to achieve $b=3$. We observe that the viral load has a steep decline but does not shoot back during the period of treatment of 48 weeks $=336$ days, and the RBC concentration is maintained at pre-treatment levels (see Figure 5 ). However, this might be clinically undesirable. 


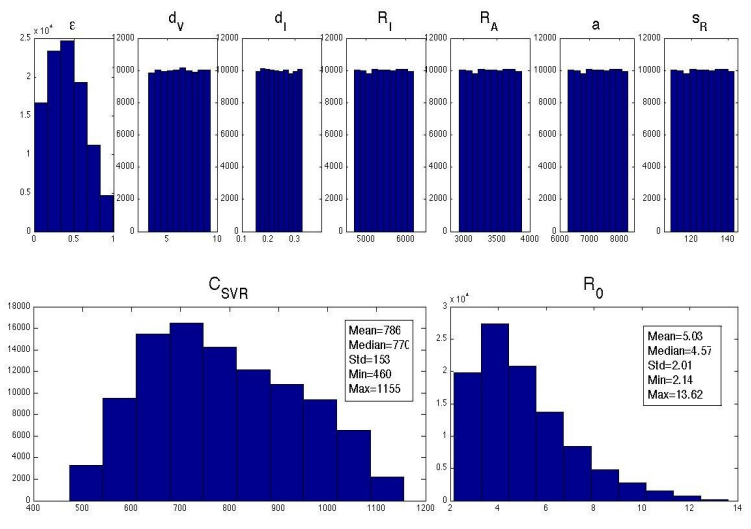

(a) Estimated distribution of $C_{S V R}$ and $R_{0}$ as functions of their parameters. The distribution of $C_{S V R}$ resembles a Gamma distribution whereas the distribution of $R_{0}$ can be approximated by a chi-square distribution.

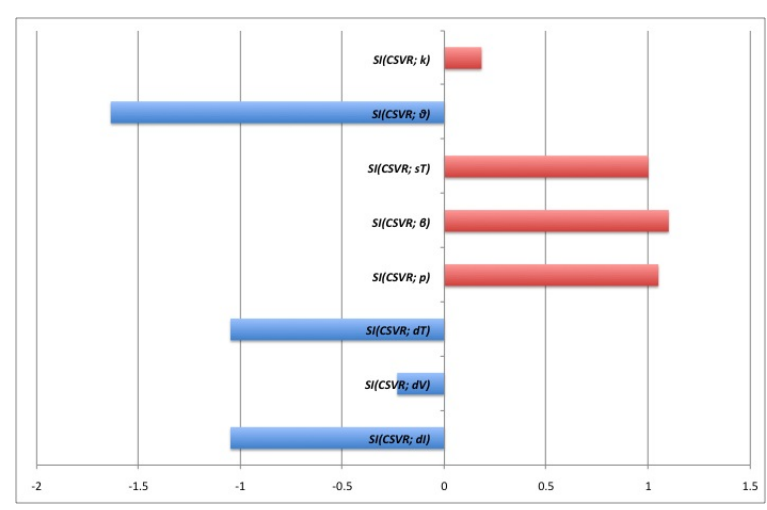

(b) Local Sensitivity Indices (SI) and relative strength of input parameters on the output variable $C_{S V R}$.

Figure 7: Results of uncertainty and sensitivity analyses

parameters will result in increases in the value of $C_{S V R}$, and the reverse for the latter set of parameters. In particular, the SI are highest for $\theta$ and $\beta$, which implies that it is critical to identify the potential effects of treatment on the rates of free virus clearance and new infections of hepatocytes, neither of which has been clearly documented to date. We therefore consider in the remainder of this section a few alternative scenarios involving these parameters.

Now, if we assume the efficacy of effect of drug on $\beta$ is $50 \%$ and compute $\alpha$ exactly like $k$, we get $\alpha$ equal to $631 \mathrm{mg}$. Keeping all the other parameters same we get the new $C_{S V R}=405 \mathrm{mg}$, from equation 15 . Then an increase in RBC production by around 1.6 times is enough to clear the virus and not cause anemia at the same time, i.e., to have $C_{A}=C_{S V R}$. We observe that changes in these parameter values can change the results significantly and thus the necessity to conduct clinical studies to estimate the mean and range of values of these parameters cannot be over-emphasized.

If we assume no effect of drug on the clearance of free $\mathrm{HCV}$ from the liver, that is, $\theta=0$, with efficacy of drug effect on $\beta$ as $50 \%$ we get $C_{S V R}=726 \mathrm{mg}$. In that case, the RBC production rate will require an increase of 2.4 times to achieve $C_{A}=C_{S V R}$.

Finally, we present in Figure 8 an illustration of the difference between administration of HCV drugs continuously or on a (discrete) daily basis. It can be seen that the amount of drug in the body under continuous dosing falls well within the sawtooth interval resulting from discrete daily dosing.

It is useful to have an estimate of the minimum increase in $\mathrm{RBC}$ production necessary to maintain healthy 

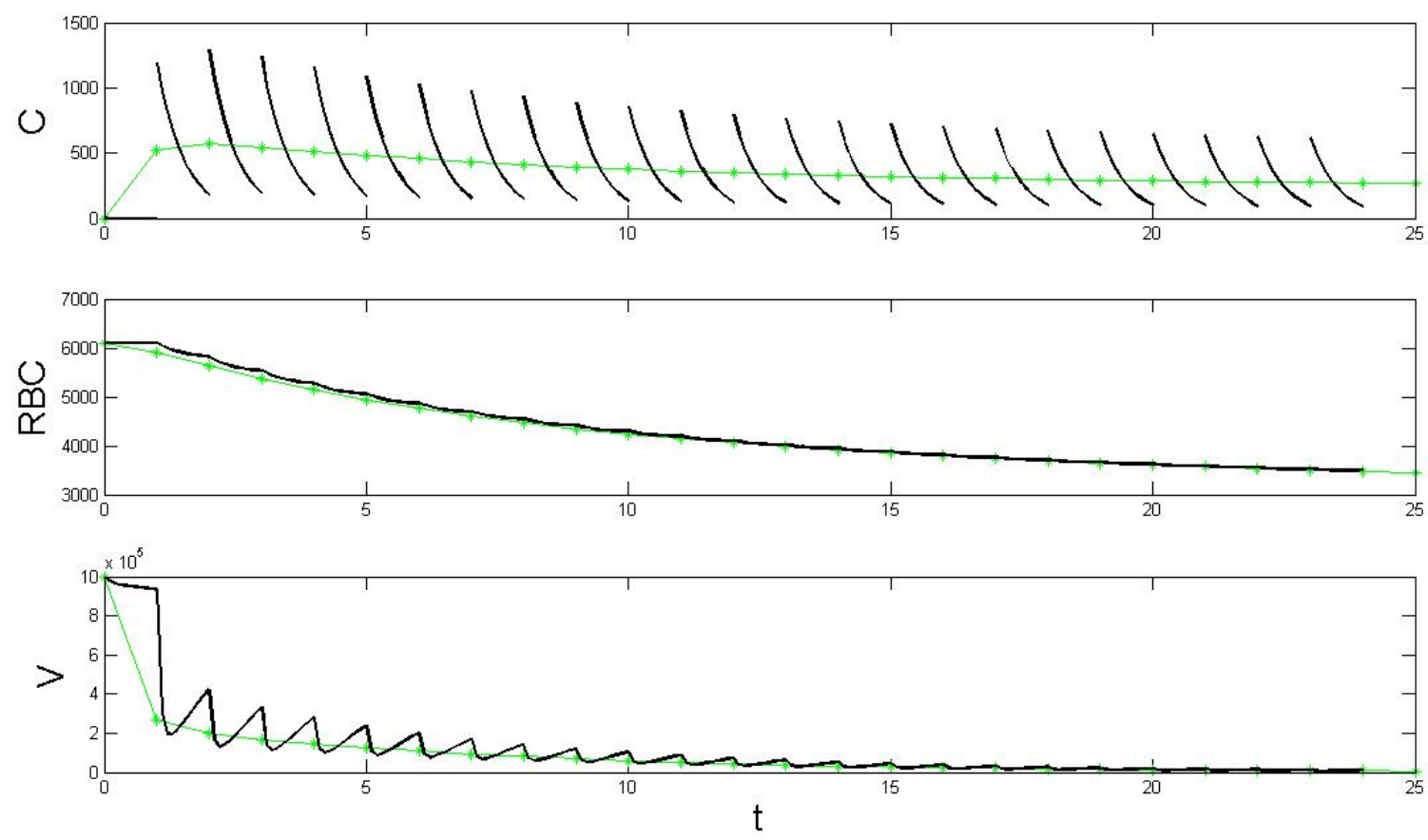

Figure 8: An illustration of the drug amount in the body, RBC level, and virion count resulting from continuous (smooth curve) vs. discrete (sawtooth) daily administration of HCV drugs during treatment. The continuous (*-) curve is generated from our model. The other curve(-) is generated from a model where the last equation is changed to $\frac{d C}{d t}=-h C$, and an impulsive dosing regimen which is adjusted and administered daily is conducted. Note total drug administered per day remains the same for the two sets of curves, as do all other model parameters.

RBC concentration above anemic levels and sufficiently reduce viral load at the same time. Choosing a responsive dosing strategy $\Delta(R)$ turns out to be pivotal, since otherwise the system would maintain the high dose irrespective of the patient's RBC level.

\section{Discussion}

Mathematical models have been used in the past to understand details of the mechanism of infection of HCV with or without treatment [18]. Models by Dixit et al. [14], Neumann et al. and Dahari et al. [7, 18] have estimated efficacies of antiviral peg-interferon by itself and in combination with ribavirin. Although this combination therapy is highly successful, it has been associated with many adverse treatment-related side effects, the most common being reversible hemolytic anemia. To combat hemolytic anemia, many patients are prescribed the drug epoietin (EPO). EPO is the artificially synthesized version of the hormone erythropoietin that stimulates RBC production in the bone marrow. Our model is extended from the immunological model 
in Neumann et al. [18] by adding two separate state variables to represent the RBC concentration and drug amount in the body as dynamical systems. Analysis of the decoupled equations separately gives two critical values of drug amount: $C^{*}$, the drug concentration required to maintain the RBC population at equilibrium, and $C_{S V R}$, the drug concentration required for patient recovery. Also, the RBC concentration and amount of drug equation helps us to calculate another critical drug amount $C_{A}$ which is the maximum amount of drug in the body that can avoid anemia.

If in a patient $C_{A}<C_{S V R}$, the doctors can prescribe a dosage to maintain the equilibrium amount of drug in the interval $\left(C_{S V R}, C_{A}\right)$ which will achieve SVR in the patient without encountering anemia. The clinical problem arises when in a patient this inequality is reversed. In that case, the doctor applies EPO to boost $C_{A}$ by increasing the RBC production to make $C_{A} \geq C_{S V R}$. The factor by which RBC needs to be increased can be calculated from our models. Our estimated set of parameters presents a 'proof of idea' situation in which this factor is found to be 2.3 to achieve $C_{A}=C_{S V R}$.

We also show through graphical simulations that a lower increase in RBC production might compromise chances of achieving SVR. Theoretically, very high doses of EPO can avoid any significant decrease in RBC concentration throughout therapy and increase tolerability of drug thus strengthening chances of achieving SVR. However, that is not practically feasible in a majority of patients. EPO has limited capabilities to increase RBC production and comes with its share of possible side effects. Thus the need to estimate the necessary increase in RBC production cannot be over-emphasized. Our model provides a way to use mathematical modeling to predict if a patient will encounter anemia under the prescribed dosing of IFN+RBV, or not. Further, it can estimate the increase in EPO-induced RBC production necessary to achieve SVR without encountering anemia.

Our model has some limitations. Firstly, we are considering a mean field model where the stochastic variability in the numerical values of parameters is not taken into account. As analyses in the previous section illustrate, the uncertainty in the estimates of several key treatment-related parameters may cause significant variations in the threshold quantities that determine patient outcomes. The parameter values for different patients with their specific strength of immune system and medical history could be varied, and it is essential to have a comprehensive notion of how dosing should be adjusted if a parameter changes within a certain range. Such a detailed understanding of the system, however, will require targeted clinical trials which can provide relevant data for fitting this model.

Second, a more detailed model could treat the two drugs, IFN and RBV, as separate state variables. 
Since they have different pharmacokinetic profiles and probably different antiviral effects, it would be a more realistic representation of the scenario. However, for that to be useful it is necessary to have well-established theories regarding the modes of action of these drugs independently and in synergy, which is not yet available [1]. Also, the initial dose of IFN and RBV for a specific patient is determined based upon his/her body mass, liver condition, etc. In this model this differentiation is implicit in taking the $\Delta_{I}$ as $1000 \mathrm{mg}$ for patients with body mass less than $75 \mathrm{~kg}$ and $1200 \mathrm{mg}$ for body mass greater than $75 \mathrm{~kg}$. The model can be made more realistic by putting in parameters or modifying initial conditions of the differential equations to take into account the intrinsic properties of a patient. However, including this change will necessitate estimation of more drug-specific parameters, and relevant patient data will be essential.

\section{Acknowledgements}

We would like to thank Dr. Carlos Castillo-Chavez and MTBI/SUMS for giving us this opportunity and, the National Science Foundation (DMS-0502349), National Security Agency (DOD-H982300710096), the Sloan Foundation, Research Initiative for Scientific Enhancement (RISE) program (GM59429) and Arizona State University for making this research possible. We thank Dr. Abdessamad Tridane for introducing us to this topic. Our heartiest appreciation goes to Dr. Maia Martcheva and Dr. Ben Bolker for their insightful suggestions. We also thank Dr. Sergei Pilyugin, Dr. Patrick DeLeenher and the reviewers for helping us improve the paper. CMKZ and AM acknowledge the support of a Norman Hackerman Advanced Research Program grant. 


\section{Appendix}

\section{A.1. Finding Equilibrium Points for $(R, C)$ system}

Equating $\frac{d C}{d t}=0$, we get $C^{*}=\frac{\lambda R^{2}}{h\left(a^{2}+R^{2}\right)}$.

Substituting $C^{*}$ into $\frac{d R}{d t}=0$, we get

$$
0=s_{R}-d_{R} R-\tau C^{*} R=s_{R}-d_{R} R-\frac{\tau \lambda R^{3}}{h\left(a^{2}+R^{2}\right)} .
$$

Multiplying by $h\left(a^{2}+R^{2}\right)$ and then dividing by $h$ results in

$$
F(R)=\left(d_{R}+\frac{\tau}{h} \lambda\right) R^{3}-s_{R} R^{2}+d_{R} a^{2} R-s_{R} a^{2} .
$$

Next, dividing by $a^{3}$ throughout, we get

$$
\left(d_{R}+\tau \frac{\lambda}{h}\right)\left(\frac{R}{a}\right)^{3}-\frac{s_{R}}{a}\left(\frac{R}{a}\right)^{2}+d_{R}\left(\frac{R}{a}\right)-\frac{s_{R}}{a}=0
$$

Again dividing throughout by $\frac{s_{R}}{a}$, we get

$$
\frac{\left(d_{R}+\tau \frac{\lambda}{h}\right)\left(\frac{R}{a}\right)^{3}}{\left(\frac{s_{R}}{a}\right)}-\left(\frac{R}{a}\right)^{2}+\frac{d_{R}}{\left(\frac{s_{R}}{a}\right)}\left(\frac{R}{a}\right)-1=0 .
$$

Now we rescale using $r=\frac{R}{a}, d=\frac{d_{R}}{\left(\frac{s_{R}}{a}\right)}, \widetilde{a}=\frac{\tau \frac{\lambda}{h}}{\left(\frac{S_{R}}{h}\right)}$. Then we get

$$
f(r)=(\widetilde{a}+d) r^{3}-r^{2}+d r-1=0 .
$$

Here note $f(0)=-1, \lim _{r \rightarrow \infty} f(r)=+\infty>0$ as $\widetilde{a}+d>0$, and most importantly $f(r)<0$ if $r \leq 0$ (because if $r \leq 0$, all terms of the equation will be $<0$ ). 


\section{A.2. Bifurcation Analysis for $(\mathbf{R}, \mathbf{C})$ system}

From the previous section we have

$$
f(r)=(\widetilde{a}+d) r^{3}-r^{2}+d r-1=0 .
$$

Now the possible cases are as follows: We know that at a bifurcation point, $f(r)=0$ and $f^{\prime}(r)=0$, since at this point the sub-system will have two positive real roots; thus for our system

$$
\begin{gathered}
f(r)=0 \Rightarrow \widetilde{a} r^{3}+d r\left(r^{2}+r\right)=r^{2}+1, \\
f^{\prime}(r)=0 \Rightarrow 3 \widetilde{a} r^{2}+d\left(3 r^{2}+1\right)=2 r .
\end{gathered}
$$

This is a linear system with respect to $\widetilde{a}$ and $d$. Hence we can write:

$$
\left[\begin{array}{cc}
r^{3} & r^{3}+r \\
3 r^{2} & 3 r^{2}+1
\end{array}\right]\left[\begin{array}{l}
\tilde{a} \\
d
\end{array}\right]=\left[\begin{array}{c}
r^{2}+1 \\
2 r
\end{array}\right] .
$$

Now, since the determinant of the coefficient matrix is non-zero $(r \neq 0)$, we can calculate its inverse to get

$$
\left[\begin{array}{c}
\tilde{a} \\
d
\end{array}\right]=\left(\frac{-1}{2 r^{3}}\right)\left[\begin{array}{cc}
3 r^{2}+1 & -r^{3}-r \\
-3 r^{2} & r^{3}
\end{array}\right]\left[\begin{array}{c}
r^{2}+1 \\
2 r
\end{array}\right] .
$$

Therefore,

$$
\begin{array}{r}
\tilde{a}=-\frac{\left(r^{2}+1\right)^{2}}{2 r^{3}}<0 . \\
d=\frac{r^{4}+3 r^{2}}{2 r^{3}}=\frac{r^{2}+3}{2 r}>0 .
\end{array}
$$

Thus our bifurcation line lies in the second quadrant of the parameter plane. However, motivated by biological reasons, our region of interest lies in the first quadrant where $\widetilde{a}>0$ and $d>0$. Plugging in $(\widetilde{a}, d)=(1,1)$ in $f(r)$ we get $f(r)=2 r^{3}-r^{2}+r-1$. In this case, $f(r)=0$ has only one positive real root. Hence, we conclude that we have only one equilibrium point throughout the first quadrant. 


\section{A.3. Local Stability of $(\mathbf{R}, \mathbf{C})$ system}

Recall that

$$
\begin{aligned}
& \frac{d R}{d t}=s_{R}-d_{R} R-\tau C R \\
& \frac{d C}{d t}=\lambda \frac{R^{2}}{\left(a^{2}+R^{2}\right)}-h C .
\end{aligned}
$$

From the previous results, we derive that

$$
J_{\left(R^{*}, C^{*}\right)}=\left[\begin{array}{cc}
-d_{R}-\tau C^{*} & -\tau R^{*} \\
2 \frac{a^{2} \lambda R^{*}}{\left(a^{2}+R^{* 2}\right)^{2}} & -h
\end{array}\right]
$$

Since $d_{R}>0, C^{*}>0, h>0, \tau>0$, we have $\Rightarrow \operatorname{tr}(J)=-\left(d_{R}+\tau C^{*}+h\right)<0$, and $\operatorname{det}(J)=$ $h\left(d_{R}+\tau C^{*}\right)+\tau R^{*} \frac{2 a^{2} R^{*} \lambda}{a^{2}+R^{* 2}}>0$

$\therefore\left(R^{*}, C^{*}\right)$ is locally asymptotically stable.

\section{A.4. Global Stability of $(R, C)$}

According to the Poincaré-Bendixson Theorem there are three possibilities for end behavior solutions to our system: a limit cycle, an unbounded solution or a globally stable equilibrium point. We use Dulac's criterion and the Poincaré-Bendixson Theorem to prove global stability of $\left(R^{*}, C^{*}\right)$ which allows us to say that $C(t)$ is asymptotically constant.

To apply Dulac's Criterion, let $g=\frac{1}{R}$. Then we have

$$
\begin{aligned}
\nabla \cdot(g \dot{x}) & =\frac{\partial}{\partial R}\left(\frac{s_{R}}{R}-d_{R} \tau C\right)+\frac{\partial}{\partial C}\left(\lambda \frac{R}{a^{2}+R^{2}}-\frac{h C}{R}\right) \\
& =-\frac{s_{R}}{R^{2}}-\frac{h}{R} \\
& <0
\end{aligned}
$$

$\therefore$ there is no limit cycle. 
We observe that

$$
\frac{d C}{d t}<\lambda-h C
$$

so $\lim \sup _{t \rightarrow \infty} C(t) \leq \frac{\lambda}{h}$. Likewise

$$
\frac{d R}{d t}<s_{R}-d_{R} R
$$

so $\lim \sup _{t \rightarrow \infty} R(t)<\frac{s_{R}}{d_{R}}$; hence the solution cannot be unbounded and by the Poincaré-Bendixson Theorem we have a globally stable equilibrium. We see that local stability has extended to global stability.

\section{A.5. Analysis of $(\mathbf{T}, \mathbf{I}, \mathbf{V})$ System}

Since $\left(R^{*}, C^{*}\right)$ is globally stable in the $(\mathrm{R}, \mathrm{C})$ system, we can apply a theorem of Thieme [19] to conclude that the dynamics of the full system (1)- (5) are asymptotic to those of the reduced (T, I, V) system (1)-(3) with $C=C^{*}$. To find the equilibria of the reduced system, we write

$$
\begin{aligned}
\frac{d T}{d t} & =s_{T}-d_{T} T-\frac{\alpha}{\alpha+C} \beta T V=0 \\
\frac{d I}{d t} & =\frac{\alpha}{\alpha+C} \beta T V-d_{I} I=0 \\
\frac{d V}{d t} & =p \frac{k}{k+C^{*}} I-\theta C V-d_{V} V=0
\end{aligned}
$$

To determine the disease free equilibrium (DFE), we let $I=0$ and $V=0$ and solve $\frac{d T}{d t}=0$ for $T$. It is clear by inspection that the DFE is $\left(\frac{s_{T}}{d_{T}}, 0,0\right)$.

\section{A.5.1. Stability Analysis of DFE}

From the previous section we know that the DFE is $\left(\frac{S_{T}}{d_{T}}, 0,0\right)$; now we will analyze the stability of the DFE. Using the Jacobian matrix,

$$
J_{\left(T^{*}, I^{*}, V^{*}\right)}=\left[\begin{array}{ccc}
-d_{T}-\frac{\alpha \beta V}{\alpha+C} & 0 & -\frac{\alpha \beta T}{\alpha+C} \\
\frac{\alpha \beta V}{\alpha+C} & -d_{I} & \frac{\alpha \beta T}{\alpha+C} \\
0 & \frac{p k}{k+C} & -\theta C-d_{V}
\end{array}\right] .
$$


Substituting $\left(\frac{s_{T}}{d_{T}}, 0,0\right)$ for $\left(T^{*}, I^{*}, V^{*}\right)$ yields

$$
J_{\left(\frac{s_{T}}{d_{T}}, 0,0\right)}=\left[\begin{array}{ccc}
-d_{T} & 0 & -\frac{\alpha \beta \frac{s_{T}}{d_{T}}}{(\alpha+C)} \\
0 & -d_{I} & \frac{\alpha \beta \frac{s_{t}}{d_{T}}}{(\alpha+C)} \\
0 & \frac{p k}{k+C} & -\theta C-d_{V}
\end{array}\right] .
$$

The characteristic equation is:

$$
\left(-d_{T}-\lambda\right)\left|\begin{array}{cc}
-d_{I}-\lambda & \frac{\alpha \beta \frac{s_{T}}{d_{T}}}{\alpha+C} \\
\frac{p k}{k+C} & -\theta C-d_{V}-\lambda
\end{array}\right|=0 .
$$

We have that $\lambda_{1}=-d_{T}^{*}$; now we consider the other sub-matrix and find its eigenvalues.

$$
\left(d_{I}+\lambda\right)\left(\theta C+d_{V}+\lambda\right)-\left(\frac{\alpha \beta \frac{s_{T}}{d_{T}}}{\alpha+C}\right)\left(\frac{p k}{k+C}\right)=0
$$

$$
\lambda^{2}+\left(d_{I}+\theta C+d_{V}\right) \lambda+d_{I}\left(\theta C+d_{V}\right)-\left(\frac{\alpha \beta \frac{s_{T}}{d_{T}}}{\alpha+C}\right)\left(\frac{p k}{k+C}\right)
$$

Let $\delta=d_{I}\left(\theta C+d_{V}\right)-\left(\frac{\alpha \beta \frac{s_{T}}{d_{T}}}{\alpha+C}\right)\left(\frac{p k}{k+C}\right)$. Now we apply the quadratic formula,

$$
\lambda_{2,3}=\frac{-\left(d_{I}+\theta C+d_{V}\right) \pm \sqrt{\left(d_{I}+\theta C+d_{V}\right)^{2}-4 \delta}}{2}<0 .
$$

For stability of the DFE, we want all the eigenvalues to have negative real parts. If $\sqrt{\left(d_{I}+\theta C+d_{V}\right)^{2}-4 \delta}$ is imaginary, then we are done as $-\left(d_{I}+\theta C+d_{V}\right)$ is always negative. If $-\left(d_{I}+\theta C+d_{V}\right)-\sqrt{\left(d_{I}+\theta C+d_{V}\right)^{2}-4 \delta}$ is real then it is negative. Thus we only consider $\lambda_{2}$, and the criterion for stability of DFE is

$$
\begin{aligned}
& -\left(d_{I}+\theta C+d_{V}\right)+\sqrt{\left(d_{I}+\theta C+d_{V}\right)^{2}-4 \delta}<0 \\
\Leftrightarrow & \left(d_{I}+\theta C+d_{V}\right)>\sqrt{\left(d_{I}+\theta C+d_{V}\right)^{2}-4 \delta} \\
\Leftrightarrow & \left(d_{I}+\theta C+d_{V}\right)^{2}>\left(d_{I}+\theta C+d_{V}\right)^{2}-4 \delta .
\end{aligned}
$$


We can cancel out like terms from both sides, since they are both positive.

$$
\begin{aligned}
& \Leftrightarrow-4 \delta<0 \\
& \Leftrightarrow \delta>0 \\
& \Leftrightarrow \quad d_{I}\left(\theta C+d_{V}\right)-\frac{\alpha \beta s_{T}}{d_{T}(\alpha+C)} \frac{p k}{k+C}>0 \\
& \Leftrightarrow \widehat{\mathfrak{R}}=\frac{\alpha \beta s_{T} p k}{d_{I} d_{T}(\alpha+C)(k+C)\left(\theta C+d_{V}\right)}<1 .
\end{aligned}
$$

Thus the DFE is locally asymptotically stable if and only if $\widehat{\mathfrak{R}}<1$.

\section{A.5.2. Endemic Equilibrium}

Now, we solve for the endemic equilibrium points, using $V^{*} \neq 0$ and $I^{*} \neq 0$.

$$
\begin{aligned}
\frac{d I}{d t} & =\frac{\alpha}{\alpha+C} \beta T V-d_{I} I=0 \\
\Rightarrow I^{*} & =\frac{\alpha}{\alpha+C} \frac{\beta T^{*} V^{*}}{d_{I}} \\
\frac{d V}{d t} & =p \frac{k}{k+C} I-\theta C V-d_{V} V=0 \\
0 & =\frac{p \alpha k \beta}{(k+C)(\alpha+C)} T^{*} V^{*}-\theta C V^{*}-d_{V} V^{*} \\
V^{*} \neq 0 \Rightarrow T^{*} & =\frac{d_{I}\left(d_{V}+\theta C\right)(k+C)(\alpha+C)}{k \alpha p \beta}
\end{aligned}
$$

From $\frac{d T}{d t}=0$, we see that

$$
\begin{aligned}
s_{T}-d_{T} T & =\frac{\alpha}{\alpha+C} \beta T V, \\
\Rightarrow V^{*} & =\frac{s_{T}}{T^{*} \beta \frac{\alpha}{\alpha+C}}-\frac{d_{T}(\alpha+C)}{\alpha \beta}, \\
\Rightarrow V^{*} & =\frac{s_{T} k p}{d_{I}\left(d_{V}+\theta C\right)(k+C)}-\frac{d_{T}(\alpha+C)}{\beta \alpha} .
\end{aligned}
$$

Thus, the endemic equilibrium is

$$
\begin{array}{r}
\left(T^{*}, I^{*}, V^{*}\right)=\left(\frac{d_{I}\left(d_{V}+\theta C\right)(k+C)(\alpha+C)}{k \alpha p \beta}, \frac{\alpha}{\alpha+C} \frac{\beta T^{*} V^{*}}{d_{I}},\right. \\
\left.\frac{s_{T} k p}{d_{I}\left(d_{V}+\theta C\right)(k+C)}-\frac{d_{T}(\alpha+C)}{\beta \alpha}\right)
\end{array}
$$


Then simplifying using $\widehat{\Re}$, the endemic equilibrium is

$$
\begin{aligned}
T^{*} & =\frac{s_{T}}{d_{T} \widehat{\Re}} \\
I^{*} & =\frac{s_{T}}{d_{I}}\left(1-\frac{1}{\widehat{\Re}}\right) \\
V^{*} & =\frac{(\alpha+C) d_{T}}{\alpha \beta}(\widehat{\Re}-1)
\end{aligned}
$$

\section{A.5.3. Local Stability of Endemic Equilibrium}

Into the Jacobian matrix,

$$
J_{\left(T^{*}, I^{*}, V^{*}\right)}=\left[\begin{array}{ccc}
-d_{T}-\frac{\alpha \beta V}{\alpha+C^{*}} & 0 & -\frac{\beta \alpha T^{*}}{\alpha+C^{*}} \\
\frac{\alpha \beta V^{*}}{\alpha+C^{*}} & -d_{I} & \frac{\alpha \beta T^{*}}{\alpha+C^{*}} \\
0 & \frac{p k}{k+C^{*}} & -\theta C^{*}-d_{V}
\end{array}\right]
$$

we substitute $\left(T^{*}, I^{*}, V^{*}\right)$ for definitions (A.33)-(A.35); the following Jacobian matrix results:

$$
\widehat{J}=\left[\begin{array}{ccc}
-d_{T}-d_{T}(\widehat{\mathfrak{R}}-1)-\lambda & 0 & -\frac{\alpha \beta s_{T}}{d_{T} \widehat{\mathfrak{R}(\alpha+C)}} \\
d_{T}(\widehat{\mathfrak{R}}-1) & -d_{I}-\lambda & \frac{\alpha \beta s_{T}}{d_{T} R(\alpha+C)} \\
0 & \frac{p k}{k+C} & -\theta C-d_{V}-\lambda
\end{array}\right]
$$

From this matrix, we determine the characteristic equation:

$$
\begin{array}{r}
P(\lambda)=-\left(d_{T} \widehat{\mathfrak{R}}+\lambda\right)\left(d_{I}+\lambda\right)\left(\theta C^{*} d_{V}+\lambda\right)+\left(d_{T} \widehat{\mathfrak{R}}+\lambda\right) \frac{\alpha \beta s_{T} p k}{d_{T}\left(\alpha+C^{*}\right)\left(k+C^{*}\right) \widehat{\Re}} \\
-d_{I} d_{T}\left(\theta C^{*}+d_{V}\right)(\widehat{\Re}-1)
\end{array}
$$

Let $q=\left(\theta C^{*}+d_{V}\right)$, then

$$
P(\lambda)=-\lambda^{3}+\lambda^{2}\left(d_{I}+d_{T} \widehat{\mathfrak{R}}+q\right)+\lambda d_{T} \widehat{\mathfrak{R}}\left(q+d_{I}\right)-d_{I} d_{T} q(1-\widehat{\mathfrak{R}})=0
$$


Now we apply the Routh-Hurwitz Criterion to determine stability. Let

$$
D=\frac{\alpha}{\alpha+C} \frac{k}{k+C} p \beta s_{T}
$$

Then we can write

$$
\widehat{\Re}=\frac{D}{d_{I} d_{T} q} .
$$

We have analyzed the disease free equilibrium to understand under which conditions the DFE is stable. Now our attention shifts to the endemic equilibrium since our biological interest is treatment of chronically HCV infected individuals. Turning to the endemic equilibrium, we solve to get:

The Routh-Hurwitz criteria are now the following conditions:

1. $\left(d_{I}+d_{T} \widehat{\mathfrak{R}}+q\right)>0$

2. $d_{I} d_{T} q(\widehat{\Re}-1)>0$

3. $\left(d_{I}+d_{T} \widehat{\mathfrak{R}}+q\right) d_{T} \widehat{\mathfrak{R}}\left(q+d_{I}\right)>-d_{I} d_{T} q(1-\widehat{\mathfrak{R}})$

Given the assumed positivity of the parameters, the first condition always holds, and the second condition is true if and only if $\widehat{\mathfrak{R}}>1$. If $\widehat{\mathfrak{R}}>1$ is true and condition 3 is satisfied then the endemic equilibrium is stable. From condition 3, we have

$$
D^{2} \frac{\left(q+d_{I}\right)}{d_{I}^{2} d_{T} q^{2}}+D\left(\frac{\left(q+d_{I}\right)^{2}}{d_{I} d_{T} q}-\frac{1}{d_{T}}\right)+d_{I} q>0
$$

Equating the left hand side of equation (A.38) to zero, we obtain two roots, $D_{1,2}$ as

$$
D_{1,2}=\frac{-\left(\frac{\left(q+d_{I}\right)^{2}}{d_{I} d_{T} q}-\frac{1}{d_{T}}\right) \pm \sqrt{\left(\frac{\left(q+d_{I}\right)^{2}}{d_{I} d_{T} q}-\frac{1}{d_{T}}\right)^{2}-4 \frac{\left(q+d_{I}\right)}{d_{I} d_{T} q}}}{2\left(\frac{q+d_{I}}{d_{I}^{2} d_{T} q^{2}}\right)}
$$

Let

$$
Q=\left(\frac{\left(q+d_{I}\right)^{2}}{d_{I} d_{T} q}-\frac{1}{d_{T}}\right)^{2}-4 \frac{\left(q+d_{I}\right)}{d_{I} d_{T} q}
$$

If $Q$ is negative, then inequality (A.38) is always satisfied. Furthermore, since $4 \frac{\left(q+d_{I}\right)}{d_{I} d_{T} q}$ is positive, if $Q$ is 
positive, then $-\left(\frac{\left(q+d_{I}\right)^{2}}{d_{I} d_{T} q}-\frac{1}{d_{T}}\right)$ dominates the sign of the roots, making $D_{1,2}<0$ since

$$
\left(\frac{\left(q+d_{I}\right)^{2}}{d_{I} d_{T} q}-\frac{1}{d_{T}}\right)>0
$$

which can be seen from the fact that

$$
\left(\frac{\left(q+d_{I}\right)^{2}}{d_{I} d_{T} q}-\frac{1}{d_{T}}\right)>0 \Leftrightarrow\left(q-d_{I}\right)^{2}>d_{I} q
$$

\section{A.5.4. Global Stability of Endemic Equilibrium}

Observation 6. The endemic equilibrium $\left(T^{*}, I^{*}, V^{*}\right)$, of the $(T, I, V)$ system is globally asymptotically stable, when it exists (i.e., $R>1$ ).

Proof. Let us define

$$
f(T)=s_{T}-d_{T} T
$$

Then,

$$
\begin{aligned}
\left(f(T)-f\left(T^{*}\right)\right)\left(1-\frac{T^{*}}{T}\right) & =-d_{T}\left(T-T^{*}\right)\left(1-\frac{T^{*}}{T}\right) \\
& =-\frac{d_{T}}{T}\left(T-T^{*}\right)^{2} \\
& \leq 0 .
\end{aligned}
$$

The above inequality holds true since $T$, the concentration of healthy hepatocytes is always positive. Recall that the endemic equilibrium is

$$
\begin{array}{r}
\left(T^{*}, I^{*}, V^{*}\right)=\left(\frac{d_{I}\left(d_{V}+\theta C\right)(k+C)(\alpha+C)}{k \alpha p \beta}, \frac{\alpha}{\alpha+C} \frac{\beta T^{*} V^{*}}{d_{I}},\right. \\
\left.\frac{s_{T} k p}{d_{I}\left(d_{V}+\theta C\right)(k+C)}-\frac{d_{T}(\alpha+C)}{\beta \alpha}\right)
\end{array}
$$


Also, at this equilibrium we get the following equalities from the equilibrium conditions:

$$
\begin{array}{r}
\frac{\alpha}{\alpha+C} \beta T^{*}=\frac{d_{I}\left(d_{V}+\theta C\right)(k+C)}{k p} \\
f\left(T^{*}\right)=\frac{\alpha}{\alpha+C} \beta T^{*} V^{*}=d_{I} I^{*} \\
\frac{p k}{k+C} I^{*}=\left(\theta C+d_{V}\right) V^{*}
\end{array}
$$

Now, let $\xi, \eta, \zeta$ be chosen constants and $W$ be the Liapunov function.

$$
W=\xi \int_{T^{*}}^{T}\left(1-\frac{T^{*}}{\tau}\right) d \tau+\eta \int_{I^{*}}^{I}\left(1-\frac{I^{*}}{\tau}\right) d \tau+\zeta \int_{V^{*}}^{V}\left(1-\frac{V^{*}}{\tau}\right) d \tau
$$

Then

$$
\begin{array}{r}
W^{\prime}=\xi\left(1-\frac{T^{*}}{T}\right)\left(s_{T}-d_{T} T-\frac{\alpha}{\alpha+C} \beta T V\right)+\eta\left(1-\frac{I^{*}}{I}\right)\left(\frac{\alpha}{\alpha+C} \beta T V-d_{I} I\right) \\
+\zeta\left(1-\frac{V^{*}}{V}\right)\left(p \frac{k}{k+C} I-\theta C V-d_{V} V\right)
\end{array}
$$

Choosing $\zeta=\xi d_{I} \frac{k+C}{p k}, \xi=\eta=1$ and using (A.44), (A.45), (A.46), (A.41) simplified $W^{\prime}$ becomes

$$
\begin{array}{r}
W^{\prime}=f(T)\left(1-\frac{T^{*}}{T}\right)-\frac{\alpha}{\alpha+C} \beta I^{*} \frac{T V}{I}+d_{I} I^{*}-d_{I} I \frac{V^{*}}{V} \\
+d_{I} \frac{k+C}{p k}\left(\theta C+d_{V}\right) V^{*}
\end{array}
$$

Then adding and subtracting $f\left(T^{*}\right)\left(1-\frac{T^{*}}{T}\right)$ yields

$$
\begin{array}{r}
W^{\prime}=\left(f(T)-f\left(T^{*}\right)\right) \\
\left(1-\frac{T^{*}}{T}\right)-\frac{\alpha}{\alpha+C} \beta I^{*} \frac{T V}{I}+d_{I} I^{*} \\
+f\left(T^{*}\right)\left(1-\frac{T^{*}}{T}\right)+d_{I} I^{*}-d_{I} I \frac{V^{*}}{V}
\end{array}
$$


Now using (A.45), (A.46) we get,

$$
W^{\prime}=\left(f(T)-f\left(T^{*}\right)\right)\left(1-\frac{T^{*}}{T}\right)-d_{I} I^{*}\left[\frac{T^{*}}{T}+\frac{T V I^{*}}{T^{*} V^{*} I}+\frac{V^{*} I}{V I^{*}}-3\right]
$$

We know from construction that the first term is negative. The second term is also negative since the geometric mean of the 3 non-negative terms $\left(\left(\frac{T^{*}}{T} \cdot \frac{T V I^{*}}{T^{*} V^{*} I} \cdot \frac{V^{*} I}{V I^{*}}\right)^{\frac{1}{3}}=1\right)$ is always less than the arithmetic mean of those terms. Therefore, $W^{\prime} \leq 0$.

We note that $W^{\prime}=0$ iff both the first and the second term is zero. The second term is zero if $\frac{T^{*}}{T}=1$ and $\frac{V^{*} I}{V I^{*}}=1$, in which case the first term also becomes zero. Thus the largest invariant set is

$\mathfrak{M}=\left\{(T, I, V) \in \operatorname{int}\left(\Re_{+}^{3}\right): T=T^{*}\right.$ and $\left.\frac{I}{I^{*}}=\frac{V}{V^{*}}\right\}$

Then LaSalle's Principle [29] implies that all bounded solutions in $\operatorname{int}\left(\Re_{+}^{3}\right)$ converge to the largest invariant set in $\mathfrak{M}$. Now to show the boundedness of the system. Consider,

$$
\frac{d(T+I)}{d t}=s_{T}-d_{T} T-d_{I} I \leq s_{T}-d_{0}(T+I)
$$

where $d_{0}=\min \left\{d_{I}, d_{T}\right\}$.

$$
\int_{T_{0}+I_{0}}^{T+I} \frac{d(T+I)}{s_{T}-d_{0}(T+I)} \leq \int_{0}^{t} d t
$$


Here, $T_{0}=T(0), I_{0}=I(0), V_{0}=V(0)$. Then

$$
\begin{aligned}
\ln \left|\frac{s_{T}-d_{0}(T+I)}{s_{T}-d_{0}\left(T_{0}+I_{0}\right)}\right| & \leq-d_{0} t \\
I+T & \leq \frac{s_{T}}{d_{0}}\left(1-e^{-d_{0} T}\right)+\left(I_{0}+T_{0}\right) e^{-d_{0} t} \\
\limsup _{t \rightarrow \infty}(I+T) & \leq \frac{s_{T}}{d_{0}}
\end{aligned}
$$

Again,

$$
\begin{aligned}
\frac{d V}{d T} & =p \frac{k}{k+C} I-\left(\theta C+d_{V}\right) V \\
& \leq p \frac{k}{k+C} \frac{s_{T}}{d_{0}}-\left(\theta C+d_{V}\right) V \\
V & \leq-\frac{e^{-\bar{t} \theta+d_{V}}}{\theta C+d_{V}}\left[\frac{p k s_{T}}{(k+C) d_{0}}-\left(\theta C+d_{V}\right) V_{0}\right]+\frac{p k s_{T}}{(K+C)\left(\theta C+d_{V}\right) d_{0}} \\
\limsup _{t \rightarrow \infty} V & \leq \frac{p k s_{T}}{(K+C)\left(\theta C+d_{V}\right) d_{0}}
\end{aligned}
$$

Hence the (T, I, V) system has bounded solutions and it is clear that the largest invariant set in $\mathfrak{M}$ is the singleton set $\left\{\left(T^{*}, I^{*}, V^{*}\right)\right\}$. This is because $\mathfrak{M}$ is a simply connected 1-dimensional domain, where solutions are always monotone. Alternatively, using $T(t)=T^{*}$ in equation 1 (i.e., $\frac{d T}{d t}=0$ ) we conclude that $\mathrm{V}$ is constant with respect to time inside $\mathfrak{M}$, which in turn implies (with equation 2) $I$ is also constant. Also, the solutions starting on the boundary move into the interior of the set except on the $T$-axis. Hence the result holds for all solutions. Therefore, $\left(T^{*}, I^{*}, V^{*}\right)$ is globally asymptotically stable.

\section{References}

[1] J. Y. N. Lau, R. C. Tam, T. J. Liang, Z. Hong, Mechanism of action of ribavirin in the combination treatment of chronic HCV infection, Hepatology 35 (5) (2005) 1002-1009.

[2] J. H. Hoofnagle, Course and outcome of hepatitis C, Hepatology 36 (5 Suppl. 1) (2002) S21-29.

[3] T. Kato, T. Date, M. Miyamoto, M. Sugiyama, Y. Tanaka, E. Orito, T. Ohno, K. Sugihara, I. Hasegawa, K. Fujiwara, K. Ito, A. Ozasa, M. Mizokami, T. Wakita, Detection of anti-hepatitis C virus effects of interferon and ribavirin by a sensitive replicon system, JCM 43 (11) (2005) 5679-5684. 
[4] C. Bain, A. Fatmi, F. Zoulim, J. P. Zarski, C. Trpo, G. Inchausp, Impaired allostimulstory function of dentric cells in chronic hepatitis C infection, Gasteroenterology.

[5] V. M. Sung, S. Shimodaira, A. L. Doughty, G. R. Picchio, H. Can, T. S. Yen, K. L. Lindsay, A. M. Levine, M. M. Lai, Establishment of B-cell lymphoma cell lines persistently infected with hepatitis C virus in vivo and in vitro: the apoptotic effects of virus infection, J. of Virol. 77 (3) (2003) 2134-2146.

[6] C. Gremion, A. Cerny, Hepatitis C virus and the immmune system: a concise review., Reviews in Medical Virology 15 (4) (2005) 235-268.

[7] H. Dahari, A. Loa, R. M. Ribeiro, A. S. Perelson, Modeling hepatitis C virus dynamics: Liver regeneration and critical drug efficacy, Journal of Theoretical Biology 247 (2007) 371-381.

[8] J. L. Dienstag, J. G. Mchutchison, American gastroenterological association medical position statement on the management of hepatitis C, Gastroenterology 130 (1) (2006) 225-230.

[9] D. B. Strader, T. Wright, D. L. Thomas, L. B. Seeff, Diagnosis, management and treatment of hepatitis C, Hepatology 39 (4) (2004) 2004.

[10] M. Sulkowski, Anemia in the treatment of hepatitis C virus infection, CID 37 (Suppl 4) (2003) S315.

[11] N. Afdhal, D. T. Dieterich, P. J. Pockros, E. R. Schiffshort, M. L. Shiffman, M. S. Sulkowski, T. Wright, Z. Younossi, B. L. Goon, K. Tang, P. Bowers, T. P. S. Group, Epoetin alpha maintains ribavirin dose in HCV-infected patients: a prospective, double-blind, randomized controlled study, Gastroenterology 126 (5) (2004) 1302-1311.

[12] U. of Sciences in Philadelphia (ed.), The Science and Practice of Pharmacy, 21st Edition, Lippincott Williams and Wilkins, Philadelphia, PA, 2006.

[13] D. T. Dieterich, R. Wasserman, N. Brau, T. I. Hassanein, E. J. Bini, P. J. Bowers, M. Mark S. Sulkowski, Once-weekly epoetin alfa improves anemia and facilitates maintenance of ribavirin dosing in hepatitis C virus infected patients receiving ribavirin plus interferon alfa, Am. J. Gastroenterol. 98 (11) (2003) $2491-2499$.

[14] N. M. Dixit, J. E. Layden-Almer, T. J. Layden, A. S. Perelson, Modelling how ribavirin improves interferon response rates in hepatitis C virus infection, Nature 432 (2004) 922-924. 
[15] A. U. Neumann, N. P. Lam, H. Dahari, M. Davidian, T. E. Wiley, B. P. Mika, A. S. Perelson, L. TJ., Differences in viral dynamics between genotypes 1 and 2 of hepatitis C virus, J. Infect. Dis 182 (2000) $28-35$.

[16] G. L. Davis, J. B. Wong, J. G. McHutchison, M. P. Manns, J. Harvey, J. Albrecht, Early virologic response to treatment with peginterferon alfa-2b plus ribavirin in patients with chronic hepatitis $\mathrm{C}$, Hepatology 38 (3) (2003) 645-652.

[17] K. A. Powers, N. M. Dixit, R. M. Ribeiro, P. Golia, A. H. Talal, A. S. Perelson, Modeling viral and drug kinetics: hepatitis C virus treatment with pegylated interferon alfa-2b., Semin. Liver Dis. 23 (Suppl 1) (2003) 13-18.

[18] U. A. Neumann, N. P. Lam, H. Dahari, D. R. Gretch, T. E. Wiley, T. J. Layden, A. S. Perelson, Hepatitis C viral dynamics in vivo and the antiviral efficacy of interferon-alpha therapy, Science 282 (2) (1998) 103-107.

[19] H. R. Thieme, Convergence results and a Poincaré Bendixson trichotomy for asymptotically autonomous differential equations, J of Math. Biol. 30 (1992) 775-763.

[20] D. S. Callaway, A. S. Perelson, HIV-1 infection and low steady state viral loads, Bull. Mathematical Biology 64 (1) (2002) 29-64.

[21] P. DeLeenheer, S. S. Pilyugin, Multi-strain virus dynamics with mutations: A global analysis, Mathematical Medicine and Biology 25 (4) (2008) 285-322.

[22] M. Mackey, Periodic auto-immune hemolytic anemia: An induced dynamical disease., Bull Math Biol 41 (1979) 829-834.

[23] L. T. Baxter, F. Yuan, R. K. Jain, Pharmacokinetics analysis of the perivascular distribution of bifuntional antibodies and haptens: Comparison with experimental data, Cancer Res. 52 (1992) 5838-5844.

[24] R. C. Davis, M. K. Davies, G. Y. H. Lip, ABC of Heart Failure, 2nd Edition, Wiley-Blackwell, 2007.

[25] S. L. Bricker, R. P. Langlais, C. S. Miller, Oral Diagnosis, Oral Medicine and Treatment Planning, 2nd Edition, BC Decker Inc, Ontario, Canada, 2002. 
[26] S. Takaki, A. Tsubota, T. H. et.al., Factors contributing to ribavirin dose reduction due to anemia during interferon alfa2b and ribavirin combination therapy for chronic hepatitis C, J Gastroenterol 39 (2004) $668-673$.

[27] H. Dahari, R. M. Ribeiro, A. S. Perelson, Triphasic decline of hepatitis C virus RNA during antiviral therapy, Hepatology 46 (2007) 16-21.

[28] E. Herrmann, J. Lee, G. Marinos, M. Modi, S. Zeuzem, Effect of ribavirin on hepatitis C viral kinetics in patients treated with pegylated interferon, Hepatology 37 (6) (2003) 1351-8.

[29] J. P. LaSalle, Stability theory for ordinary differential equations, J. Diff. Eqns. 4 (1968) 57-65. 